\title{
REVISION OF PARASITIC HELMINTHS REPORTED IN FRESHWATER FISH FROM TURKEY WITH NEW RECORDS
}

Ahmet ÖKTENER *

* Directorate of Agricultural Research and Policy, Livestock Research Station, Department of Fisheries, Çanakkale Street km 7, Bandirma, Balikesir, Turkey, TR-10200, ahmetoktener@yahoo.com

DOI: 10.1515/trser-2015-0001

KEYWORDS: Turkey, freshwater, checklist, revision, helminths, fish.

\section{ABSTRACT}

This new checklist is an update of helminths of freshwater fish from Turkey. The last publication of a checklist of helminth parasites of freshwater fish in Turkey was over 11 years ago (Öktener, 2003), and there have been a number of new records. This update includes additional records and allows for the correction of errors and omissions that were present in the preceding version. The revision literature indicated the occurrence of 123 parasite species which included 60 monogeneans, 20 digeneans, 20 cestodes, 11 nematodes, seven acanthocephalans, five annelids from 71 different wild fish (64 native, four transitional, three introduced fish) species from freshwater in Turkey. Parasites not identified to species level are listed separately, and not included in the resulting comments, because of reporting different host species. Cyprinidae, with 50 species, is the dominant family among the examined fish with regard to species diversity.

RESUMEN: Revisión de parásitos helmintos reportados en peces de aguadulce en Turquía, con nuevos registros.

Se realizó una actualización del elenco de especies de helmintos parásitos de peces de agua dulce de Turquía. Desde la publicación del último listado de parásitos helmintos de peces dulceacuícolas, hace 11 años (Öktener, 2003), han habido cantidad de nuevos registros. Esta actualización incluye a dichos registros, lo cual permitió la corrección de aquellos errores y omisiones que se cometieron en la versión anterior del listado. La literatura revisada indica la ocurrencia de 123 especies parásitas que incluyen 60 monogeneos, 20 digeneos, 20 céstodos, 11 nemátodos, siete acantocéfalos y cinco anélidos colectados en 71 especies de peces de agua dulce en Turquía (64 especies nativas, cuatro transicionales y tres introducidas). Los parásitos no identificados a nivel especie se enlistan separadamente y no se comentan en los resultados, debido a que son reportados en distintas especies de hospederos. Los ciprínidos, con 50 especies, es la familia dominante entre los peces examinados en cuanto a diversidad específica.

REZUMAT: Revizuire a viermilor paraziți raportați la specii de pești de apă dulce din Turcia, cu noi înregistrări.

Această listă nouă de specii este rezultatul revizuirii parazitării helmintice la speciile de pești de apă dulce din Turcia. De la publicarea primei versiuni în urmă cu peste 11 ani (Öktener, 2003), au mai fost semnalate și alte specii. Prezenta versiune include și semnalările ulterioare și permite corectarea erorilor și omisiunilor din prima versiune. Literatura utilizată a indicat prezența a 123 de specii parazite care au inclus 60 specii monogenee, 20 specii de digenee, 20 de cestode, 11 nematode, șapte acanthocephali, cinci annelide, iar speciile gazdă sunt în număr de 71 (specii sălbatice dulcicole) din care 64 sunt pești autohtoni, patru de tranzit și trei specii introduse. Paraziții care nu au fost identificați la nivel de specie sunt indicați într-o listă separată fără a include comentarii, deoarece se raportează la specii gazdă diferite. Din punct de vedere al diversității speciilor studiate, familia de pești dominantă este cea a Cyprinidaelor cu 50 de specii investigate parazitologic. 


\section{INTRODUCTION}

The fish-parasite checklist studies are important taxonomic documents in order to obtain the fish-parasite relationships, host selectivity and geographic distribution of fish parasites. They may contribute as a baseline of data in the disciplines of parasitology, zoology, medicine, environmental science in terms of determining biological diversity, treatment and control of parasites, identification of parasite, determining host selectivity and geographic distribution of fish zoonoses, compare of fish parasite fauna of local, regional and worldwide. Research about the helminth parasites of freshwater fish in the world has increased in recent years (Holland and Kennedy, 1997; Arthur and Lumanlan-Mayo, 1997; Palm et al., 1999; Arthur and Ahmet, 2002; Kohn et al., 2006; Salgado-Maldanado, 2006; Luque and Tavares, 2007; Violante-González et al., 2007; Pazooki and Masoumian, 2012).

Turkey is geographically divided into seven regions: the Marmara Region, the Mediterranean Region, the Black Sea Region, the Aegean Region, the Central Anatolia Region, the Eastern Anatolian Region, and the South-Eastern Anatolian Region. These regions have 200 lakes, 206 dam lakes, 953 pond lakes and 33 rivers (Kiliç, 1999). The examination of literature on Turkish freshwater has revealed the report of 248 species (plus 13 introduced) valid freshwater fish species (Frick et al., 2007).

The parasites of Turkey's freshwater fish were poorly known until Paperna (1964) reported the cestode Caryophyllaeus brachycollis Janiszewska, in 1951 Cyprinus carpio. Articles in different journals, MSc and PhD thesis, symposium, conference proceedings and other reports have been published mentioning parasites of wild, farmed and imported fish by Turkish researchers between 1964 and 2014.

All information about parasites of freshwater fish-parasite have been compiled by Öktener (2003), based on a parasite-host list and host-parasite list, respectively. The author compiled a critical checklist of the metazoan parasites (99 helminths, 12 parasitic crustaceans, one mollusca larvae) of freshwater fish from Turkey. After this checklist, several studies and theses were published by Turkish and foreign scientists. These studies yielded some new distributional records and added new species.

This new checklist was done to update the helminths of freshwater fishes from Turkey. Finally, it was also planned to show and update the parasite richness of fishes of Turkey. It was felt that a critical summary of the freshwater fish parasites known from Turkey to date would help to solve contradictions among researchers, and benefit veterinarians, parasitologists, etc.

\section{MATERIALS AND METHODS}

Information from all available references on helminths of freshwater fishes in Turkey (journal publications, reports of research projects, thesis, proceedings of congress, symposium proceedings) from 1964 to 2014 were gathered to provide host-parasite and parasite-host lists.

In the literature dealing with the parasites of freshwater fishes in Turkey, appear many incorrect spellings of parasite names, host names and species author's names, and incorrect attributions of dates of species authorship. The scientific names of all parasites and their synonyms were checked with main electronic sites concerning with the classification (ITIS, 2013; WoRMS Editorial Board, 2014; Gibson et al., 2005). Erroneous spellings of parasite names have been consistently applied, these were noted (Tab. 1).

The scientific names of fishes were checked according to Frick et al. (2007) and the electronic sites Froese and Pauly (2013); WoRMS Editorial Board (2014); Eschmeyer (2014). Similarly, misspellings of host species names, with few exceptions where these have been widely applied, have been corrected without comment using information obtained from Froese and Pauly (2013) (Tab. 2). 
Table 1: Change of current valid names and synonymies of helminth species.

\begin{tabular}{|l|l|}
\hline \multicolumn{1}{|c|}{ Synonyms and incorrect spellings } & \multicolumn{1}{c|}{ Valid Name } \\
\hline $\begin{array}{l}\text { Diplozoon megan } \\
\text { (Nordmann, 1832) }\end{array}$ & Paradiplozoon megan \\
Silurodiscoides vistulensis & Bykhovskii and Nagibi, 1959 \\
Gussev, 1985 & Sivalodiscoides vistulensis \\
\hline Silurodiscoides siluri & Ancylodiscoides siluri \\
Gussev, 1976 & Zandt, 1924 \\
\hline Philometra abdominalis & Philometra ovate \\
Nybelin, 1928 & (Zeder, 1803) \\
\hline Bothriocephalus gowkonensis & Bothriocephalus acheilognathi \\
(Korting, 1976) & Yamaguti, 1955 \\
\hline Caryophyllaeus mutabilis & Caryophyllaeus laticeps \\
Rudolphi, 1802 & (Müller, 1781) \\
\hline Helobdella stagnates & Helobdella stagnalis \\
Linnaeus, 1758 & (Linnaeus, 1758) \\
\hline
\end{tabular}

Table 2: Change of current valid names and synonymies of fish species.

\begin{tabular}{|l|l|}
\hline \multicolumn{1}{|c|}{ Synonyms and incorrect spellings } & \multicolumn{1}{c|}{ Valid Names } \\
\hline Carassius auratus auratus & Carassius auratus \\
\hline Gobius fluviatilis & Neogobius fluviatilis \\
\hline Cobitis simplicispinna & Cobitis simplicispina \\
\hline Chondostroma regium & Chondrostoma regium \\
\hline Leuciscus cephalus & Squalius cephalus \\
\hline Leuciscus cephalus orientalis & Squalius cephalus \\
\hline Vimba vimba tenella & Vimba vimba \\
\hline Chalcalburnus chalcoides & Alburnus chalcoides \\
\hline Rhodeus sericeus amarus & Rhodeus amarus \\
\hline Rhodeus sericeus & Rhodeus amarus \\
\hline Clarias lazera & Clarias gariepinus \\
\hline Stizostedion lucioperca & Sander lucioperca \\
\hline Nemachilus sp. & Nemacheilus sp. \\
\hline Cyprinion macrostomus & Cyprinion macrostomum \\
\hline Leuciscus lepidus & Squalius lepidus \\
\hline Garra rufa obtusa & Garra rufa \\
\hline Capoeta capoeta bergamae & Capoeta bergamae \\
\hline Barbus plebejus escherichii & Luciobarbus escherichii \\
\hline Barbus rajanorum mystaceus & Luciobarbus mystaceus \\
\hline Barbus rajanorum & Luciobarbus pectoralis \\
\hline Capoeta capoeta umbla & Capoeta umbla \\
\hline Barbus capito pectoralis & Luciobarbus pectoralis \\
\hline Barbus esocinus & Luciobarbus esocinus \\
\hline Pseudophoxinus battalgil & Pseudophoxinus battalgilae \\
\hline
\end{tabular}




\section{RESULTS AND DISCUSSION}

This helminth checklist of freshwater fish from Turkey includes only Monogenea, Digenea, Cestoda, Nematoda, Acanthocephala, Annelida and it was compiled with the parasite species arranged by phylum, class and alphabetical, as appropriate. The host-parasite list/parasite-host list are arranged: (1) a list of collected parasite groups and their parasitized fish, the collection site, and author (Tab. 3); (2) a list of parasitized fish with the corresponding species of parasites (Tab. 4).

Table 3: Helminth - Host List.

\begin{tabular}{|l|l|l|}
\hline Phylum Platyhelmintes & & \\
\hline Class Monogenea & & \\
\hline $\begin{array}{l}\text { Ancylodiscoides siluri } \\
\text { Zandt, 1924 }\end{array}$ & & \\
\hline Silurus glanis & Sapanca Lake & \\
\hline Silurus glanis & İznik Lake & Soylu (1990) \\
\hline Silurus glanis & Terkos Lake & Aydoğdu et al. (1996b) \\
\hline Silurus glanis & Almus Dam Lake & Soylu (2005) \\
\hline Silurus glanis & Siğirci Lake & Turgut (2005) \\
\hline & & Çolak (2013) \\
\hline $\begin{array}{l}\text { Ancylodiscoides vistulensis } \\
\text { Sivak, 1932 }\end{array}$ & & \\
\hline Silurus glanis & & \\
\hline Silurus glanis & Sapanca Lake & \\
\hline Silurus glanis & Terkos Lake & Soylu (1990) \\
\hline Silurus glanis & Almus Dam Lake & Soylu (2005) \\
\hline & Siğirci Lake & Turgut (2005) \\
\hline $\begin{array}{l}\text { Ancyrocephalus paradoxus } \\
\text { Creplin, 1839 }\end{array}$ & & Çolak (2013) \\
\hline Sander lucioperca & & \\
\hline & Siğirci Lake & \\
\hline $\begin{array}{l}\text { Dactylogyrus affinis } \\
\text { Bychowsky, 1933 }\end{array}$ & & Çolak (2013) \\
\hline Capoeta umbla & & \\
\hline & Hazar Lake & \\
\hline $\begin{array}{l}\text { Dactylogyrus alatus } \\
\text { Linstow, 1878 }\end{array}$ & & Özgül (2008) \\
\hline Alburnus alburnus & Alo05) \\
\hline Chondrostoma regium & Almus Dam Lake & \\
\hline Alburnus alburnus & Mustafakemalpaşa Stream & \\
\hline & & \\
\hline Chondostroma regium & Murat River & \\
\hline Alburnus heckeli & & \\
\hline & & \\
\hline & & \\
\hline
\end{tabular}


Table 3 (continuing): Helminth - Host List.

\begin{tabular}{|c|c|c|}
\hline $\begin{array}{l}\text { Dactylogyrus anchoratus } \\
\text { (Dujardin, 1845) }\end{array}$ & & \\
\hline Cyprinus carpio & Bekteşağa Pond & Özer (1995) \\
\hline Carassius carassius & Enne Dam Lake & Koyun (2001) \\
\hline Carassius auratus & Enne Dam Lake & Koyun (2001) \\
\hline Carassius carassius & Kovada Lake & Özan and Kir (2005) \\
\hline Carassius gibelio & Seyitler Dam Lake & Öztürk (2010) \\
\hline Cyprinus carpio & Karacaören II Dam Lake & Samanci (2011) \\
\hline Carassius carassius & Karacaören II Dam Lake & Samanci (2011) \\
\hline Carassius auratus & Emre Dam Lake & Öztürk (2011) \\
\hline Carassius gibelio & Emre Dam Lake & Öztürk (2011) \\
\hline Carassius gibelio & Siğirci Lake & Çolak (2013) \\
\hline $\begin{array}{l}\text { Dactylogyrus ancylostylus } \\
\text { Linstow, } 1878\end{array}$ & & \\
\hline Luciobarbus pectoralis & Keban Dam Lake & Sağlam (1992) \\
\hline Luciobarbus esocinus & Keban Dam Lake & Sağlam (1992) \\
\hline Cyprinus carpio & Keban Dam Lake & Sağlam (1992) \\
\hline $\begin{array}{l}\text { Dactylogyrus asper } \\
\text { Linstow, } 1878\end{array}$ & & \\
\hline Luciobarbus esocinus & Keban Dam Lake & Sağlam (1992) \\
\hline Luciobarbus mystaceus & Keban Dam Lake & Sağlam (1992) \\
\hline $\begin{array}{l}\text { Dactylogyrus auriculatus } \\
\text { (Nordmann, 1832) }\end{array}$ & & \\
\hline Capoeta umbla & Hazar Lake & Aksoy et al. (2006) \\
\hline $\begin{array}{l}\text { Dactylogyrus baueri } \\
\text { Gussev, } 1955\end{array}$ & & \\
\hline Carassius gibelio & Siğirci Lake & Çolak (2013) \\
\hline $\begin{array}{l}\text { Dactylogyrus bicornis } \\
\text { Malevitskaya, } 1941\end{array}$ & & \\
\hline Rhodeus amarus & Sapanca, Terkos Lakes & Soylu (2009) \\
\hline $\begin{array}{l}\text { Dactylogyrus carpathicus } \\
\text { Zakhvatkin, } 1951\end{array}$ & & \\
\hline Luciobarbus escherichii & Doganci Dam Lake & Aydoğdu (2001) \\
\hline
\end{tabular}


Table 3 (continuing): Helminth - Host List.

\begin{tabular}{|c|c|c|}
\hline $\begin{array}{l}\text { Dactylogyrus chalcalburni } \\
\text { Dogiel et Bychow., } 1833\end{array}$ & & \\
\hline Alburnus chalcoides & Manyas Lake & Öztürk (2000) \\
\hline Alburnus chalcoides & Terkos Lake & Soylu (2009) \\
\hline $\begin{array}{l}\text { Dactylogyrus cornu } \\
\text { Linstow, } 1878\end{array}$ & & \\
\hline Blicca bjoerkna & Sapanca Lake & Soylu (1990) \\
\hline Vimba vimba & Manyas Lake & Öztürk (2000) \\
\hline Vimba vimba & Sirakaraağaçlar Stream & Özer and Öztürk (2005) \\
\hline Vimba vimba & Sapanca Lake & Uzunay (2006) \\
\hline $\begin{array}{l}\text { Dactylogyrus cornoides } \\
\text { (Gläser and Gussev, 1971) }\end{array}$ & & \\
\hline Vimba vimba & Sapanca Lake & Uzunay (2006) \\
\hline Blicca bjoerkna & Sapanca Lake & Soylu (2012) \\
\hline $\begin{array}{l}\text { Dactylogyrus crucifer } \\
\text { Wagener, } 1857\end{array}$ & & \\
\hline Rutilus rutilus & Sapanca Lake & Soylu (1990) \\
\hline Blicca bjoerkna & Sapanca Lake & Soylu (1990) \\
\hline Rutilus frisii & İznik Lake & Aydoğdu et al. (1997b) \\
\hline Rutilus rutilus & Manyas Lake & Öztürk (2000) \\
\hline Capoeta tinca & Almus Dam Lake & Turgut (2005) \\
\hline Rutilus rutilus & Kocadere Stream & Selver (2008) \\
\hline Capoeta tinca & Almus Dam Lake & Özgül (2008) \\
\hline Rutilus rutilus & Siğirci Lake & Çolak (2013) \\
\hline $\begin{array}{l}\text { Dactylogyrus difformis } \\
\text { Wagener, } 1857\end{array}$ & & \\
\hline $\begin{array}{l}\text { Scardinius } \\
\text { erythrophthalmus }\end{array}$ & Sapanca Lake & Soylu (1990) \\
\hline $\begin{array}{l}\text { Scardinius } \\
\text { erythrophthalmus }\end{array}$ & Manyas Lake & Öztürk (2000) \\
\hline $\begin{array}{l}\text { Scardinius } \\
\text { erythrophthalmus }\end{array}$ & Karacabey Lagoon Lake & Öztürk et al. (2002) \\
\hline $\begin{array}{l}\text { Scardinius } \\
\text { erythrophthalmus }\end{array}$ & Kocadere Stream & Selver (2008) \\
\hline $\begin{array}{l}\text { Scardinius } \\
\text { erythrophthalmus }\end{array}$ & Terkos Lake & Soylu (2009) \\
\hline $\begin{array}{l}\text { Scardinius } \\
\text { erythrophthalmus }\end{array}$ & Siğirci Lake & Çolak (2013) \\
\hline
\end{tabular}


Table 3 (continuing): Helminth - Host List.

\begin{tabular}{|c|c|c|}
\hline $\begin{array}{l}\text { Dactylogyrus difformoides } \\
\text { Glaser and Gusev, } 1967\end{array}$ & & \\
\hline $\begin{array}{l}\text { Scardinius } \\
\text { erythrophthalmus }\end{array}$ & Terkos Lake & Kahveci (2004) \\
\hline $\begin{array}{l}\text { Scardinius } \\
\text { erythrophthalmus }\end{array}$ & Sapanca Lake & Soylu (2009) \\
\hline Blicca bjoerkna & Sapanca Lake & Soylu (2012) \\
\hline $\begin{array}{l}\text { Scardinius } \\
\text { erythrophthalmus }\end{array}$ & Siğirci Lake & Çolak (2013) \\
\hline $\begin{array}{l}\text { Dactylogyrus distinguendus } \\
\text { (Linstow, 1878) }\end{array}$ & & \\
\hline Abramis brama & Terkos Lake & Karatoy (2004) \\
\hline Blicca bjoerkna & Kocadere Stream & Selver (2008) \\
\hline Blicca bjoerkna & Sapanca Lake & Soylu (2012) \\
\hline $\begin{array}{l}\text { Dactylogyrus elegantis } \\
\text { Gusev, } 1966\end{array}$ & & \\
\hline Chondrostoma regium & Murat River & Koyun (2011) \\
\hline Squalius cephalus & Murat River & Koyun (2011) \\
\hline $\begin{array}{l}\text { Dactylogyrus ergensi } \\
\text { Molnar, } 1964\end{array}$ & & \\
\hline Pseudophoxinus antalyae & $\begin{array}{l}\text { Kepez I Hydro Electric } \\
\text { Power Plant Loading Pond }\end{array}$ & Soylu and Emre (2007) \\
\hline $\begin{array}{l}\text { Dactylogyrus extensus } \\
\text { Mueller and Cleave, } 1932\end{array}$ & & \\
\hline Luciobarbus mystaceus & Keban Dam Lake & Sağlam (1992) \\
\hline Chondrostoma regium & Keban Dam Lake & Sağlam (1992) \\
\hline Cyprinus carpio & Bekteşağa Pond & Özer (1995) \\
\hline Cyprinus carpio & Uluabat Lake & Oğuz et al. (1996a) \\
\hline Cyprinus carpio & İznik Lake & Aydoğdu (1997) \\
\hline Cyprinus carpio & Manyas Lake & Öztürk (2000) \\
\hline Cyprinus carpio & Doğanci Dam Lake & Aydoğdu (2001) \\
\hline Cyprinus carpio & Karacabey Lagoon Lake & Aydoğdu et al. (2001a) \\
\hline Cyprinus carpio & Karamik Lake & Kutlu (2005) \\
\hline Cyprinus carpio & Eber Lake & Öztürk (2005) \\
\hline Cyprinus carpio & Almus Dam Lake & Turgut (2005) \\
\hline Capoeta umbla & Hazar Lake & Aksoy et al. (2006) \\
\hline Cyprinus carpio & Hazar Lake & Aksoy et al. (2006) \\
\hline Squalius cephalus & Hazar Lake & Aksoy et al. (2006) \\
\hline Cyprinus carpio & Sapanca Lake & Uzunay (2006) \\
\hline Barbus grypus & Atatürk Dam Lake & Dal (2006) \\
\hline
\end{tabular}


Table 3 (continuing): Helminth - Host List.

\begin{tabular}{|c|c|c|}
\hline Cyprinus carpio & Akşehir Lake & Kartal (2006) \\
\hline Cyprinus carpio & Selevir Dam Lake & Öztürk and Bulut (2006) \\
\hline Cyprinus carpio & $\begin{array}{l}\text { Kepez I Hydro Electric } \\
\text { Power Plant Loading Pond }\end{array}$ & Soylu and Emre (2007) \\
\hline Cyprinus carpio & Terkos Lake & Soylu (2009) \\
\hline Cyprinus carpio & Emre Dam Lake & Öztürk (2011) \\
\hline Cyprinus carpio & Tahtali Dam Lake & Karakişi and Demir (2012) \\
\hline Carassius gibelio & Siğirci Lake & Çolak (2013) \\
\hline \multicolumn{3}{|l|}{$\begin{array}{l}\text { Dactylogyrus falcatus } \\
\text { (Wedl, 1857) }\end{array}$} \\
\hline Blicca bjoerkna & Sapanca Lake & Soylu (2012) \\
\hline \multicolumn{3}{|l|}{$\begin{array}{l}\text { Dactylogyrus folkmanovae } \\
\text { Ergens, } 1956\end{array}$} \\
\hline Squalius cephalus & Doganci Dam Lake & Aydoğdu (2001) \\
\hline Squalius cephalus & Susurluk River & Gürkan and Özan (2012) \\
\hline \multicolumn{3}{|l|}{$\begin{array}{l}\text { Dactylogyrus fraternus } \\
\text { Wagener, } 1909\end{array}$} \\
\hline Alburnus alburnus & Enne Dam Lake & Koyun (2001) \\
\hline Alburnus alburnus & Mustafakemalpaşa Stream & Aydoğdu and Selver (2006) \\
\hline \multicolumn{3}{|l|}{$\begin{array}{l}\text { Dactylogyrus frisii } \\
\text { Bychowsky, } 1933\end{array}$} \\
\hline Rutilus frisii & Terkos Lake & Soylu (2009) \\
\hline \multicolumn{3}{|l|}{$\begin{array}{l}\text { Dactylogyrus inexpectatus } \\
\text { Izjumova, } 1955\end{array}$} \\
\hline Carassius gibelio & Siğirci Lake & Çolak (2013) \\
\hline \multicolumn{3}{|l|}{$\begin{array}{l}\text { Dactylogyrus izjumovae } \\
\text { Gusev, } 1966\end{array}$} \\
\hline Scardinius erythrophthalmus & Terkos Lake & Kahveci (2004) \\
\hline \multicolumn{3}{|l|}{$\begin{array}{l}\text { Dactylogyrus malleus } \\
\text { Linstow, } 1877\end{array}$} \\
\hline Barbus plebejus & Almus Dam Lake & Turgut (2005) \\
\hline Capoeta umbla & Hazar Lake & Aksoy et al. (2006) \\
\hline \multicolumn{3}{|l|}{$\begin{array}{l}\text { Dactylogyrus macrocanthus } \\
\text { Wagener, } 1857\end{array}$} \\
\hline Tinca tinca & Sapanca Lake & Soylu (1990) \\
\hline Tinca tinca & Uluabat Lake & Öztürk (2002) \\
\hline Tinca tinca & Terkos Lake & Soylu (2003) \\
\hline
\end{tabular}


Table 3 (continuing): Helminth - Host List.

\begin{tabular}{|c|c|c|}
\hline \multicolumn{3}{|l|}{$\begin{array}{l}\text { Dactylogyrus minutus } \\
\text { Kulwiec, } 1927\end{array}$} \\
\hline Luciobarbus pectoralis & Keban Dam Lake & Sağlam (1992) \\
\hline Cyprinus carpio & Keban Dam Lake & Sağlam (1992) \\
\hline Cyprinus carpio & Karacaören I Dam Lake & Kir (1998) \\
\hline Cyprinus carpio & Beyşehir Lake & Özan (2005) \\
\hline Carassius carassius & Kovada Lake & Özan and Kir (2005) \\
\hline Capoeta umbla & Hazar Lake & Aksoy et al. (2006) \\
\hline Cyprinus carpio & Kovada Lake & Kir and Özan (2007) \\
\hline \multicolumn{3}{|c|}{$\begin{array}{l}\text { Dactylogyrus naviculoides } \\
\text { Ergens, } 1960\end{array}$} \\
\hline Squalius cephalus & Almus Dam Lake & Turgut (2005) \\
\hline \multicolumn{3}{|l|}{$\begin{array}{l}\text { Dactylogyrus nybelini } \\
\text { Markewitsch, } 1933\end{array}$} \\
\hline Rutilus frisii & Terkos Lake & Soylu (2009) \\
\hline \multicolumn{3}{|l|}{$\begin{array}{l}\text { Dactylogyrus phoxini } \\
\text { (Malevitskaya, 1949) }\end{array}$} \\
\hline Cyprinus carpio & Sapanca Lake & Uzunay (2006) \\
\hline \multicolumn{3}{|l|}{$\begin{array}{l}\text { Dactylogyrus prostae } \\
\text { Molnar, } 1964\end{array}$} \\
\hline Squalius cephalus & Murat River & Koyun (2011) \\
\hline \multicolumn{3}{|l|}{$\begin{array}{l}\text { Dactylogyrus pulcher } \\
\text { Bychowsky, } 1957\end{array}$} \\
\hline Capoeta capoeta & Almus Dam Lake & Turgut (2005) \\
\hline \multicolumn{3}{|c|}{$\begin{array}{l}\text { Dactylogyrus rectotrabus } \\
\text { Gussev, Jalal and Molnar, } \\
1993\end{array}$} \\
\hline Garra rufa & Murat River & Koyun (2011) \\
\hline \multicolumn{3}{|l|}{$\begin{array}{l}\text { Dactylogyrus sphyrna } \\
\text { Linstow, } 1828\end{array}$} \\
\hline Rutilus rutilus & Sapanca Lake & Soylu (1990) \\
\hline Blicca bjoerkna & Sapanca Lake & Soylu (1990) \\
\hline Capoeta trutta & Keban Dam Lake & Sağlam (1992) \\
\hline Chondrostoma regium & Keban Dam Lake & Sağlam (1992) \\
\hline Blicca bjoerkna & Uluabat Lake & Akinci (1999) \\
\hline Blicca bjoerkna & Manyas Lake & Öztürk (2000) \\
\hline Rutilus rubilio & İznik Lake & Aydoğdu et al. (2000) \\
\hline
\end{tabular}


Table 3 (continuing): Helminth - Host List.

\begin{tabular}{|c|c|c|}
\hline Abramis brama & Terkos Lake & Karatoy (2004) \\
\hline Vimba vimba & Sapanca Lake & Uzunay (2006) \\
\hline Pseudophoxinus antalyae & $\begin{array}{l}\text { Kepez I Hydro Electric } \\
\text { Power Plant Loading Pond }\end{array}$ & Soylu and Emre (2007) \\
\hline Blicca bjoerkna & Kocadere Stream & Selver (2008) \\
\hline Vimba vimba & Gölbaşi Dam Lake & Aydoğdu et al. (2008) \\
\hline $\begin{array}{l}\text { Scardinius } \\
\text { erythrophthalmus }\end{array}$ & Sapanca Lake & Soylu (2009) \\
\hline Rutilus rutilus & Siğirci Lake & Çolak (2013) \\
\hline \multicolumn{3}{|l|}{$\begin{array}{l}\text { Dactylogyrus vastator } \\
\text { (Nybelin, 1924) }\end{array}$} \\
\hline Cyprinus carpio & Bafa Lake & Geldiay and Balik (1974) \\
\hline Aphanius sp. & Cigli Stream & Geldiay and Balik (1974) \\
\hline Capoeta capoeta & Balikliag Stream & Cengizler and Göksu (1994) \\
\hline Alburnus orontis & Balikliag Stream & Cengizler and Göksu (1994) \\
\hline Cyprinus carpio & Bekteşağa Pond & Özer (1995) \\
\hline Cyprinus carpio & Seyhan River & Cengizler et al. (2001) \\
\hline Carassius carassius & $\begin{array}{l}\text { Kepez I Hydro Electric } \\
\text { Power Plant Loading Pond }\end{array}$ & Soylu and Emre (2005) \\
\hline Silurus glanis & Yamula Dam Lake & Kilinçaslan (2007) \\
\hline Cyprinus carpio & Adana DSI & Güleç and Şahan (2010) \\
\hline Carassius gibelio & Siğirci Lake & Çolak (2013) \\
\hline \multicolumn{3}{|l|}{$\begin{array}{l}\text { Dactylogyrus vistulae } \\
\text { Prost, } 1957\end{array}$} \\
\hline Squalius cephalus & Doganci Dam Lake & Aydoğdu (2001) \\
\hline Squalius cephalus & Almus Dam Lake & Turgut (2005) \\
\hline Chondrostoma regium & Almus Dam Lake & Turgut (2005) \\
\hline Rutilus rutilus & Sapanca Lake & Karabiber (2006) \\
\hline Squalius cephalus & Örenler Dam Lake & Kurupinar (2009) \\
\hline Squalius cephalus & Susurluk River & Gürkan and Özan (2012) \\
\hline Chondrostoma regium & Murat River & Koyun (2011) \\
\hline Squalius cephalus & Murat River & Koyun (2011) \\
\hline \multicolumn{3}{|l|}{$\begin{array}{l}\text { Dactylogyrus wunderi } \\
\text { Bykhovskii, } 1931\end{array}$} \\
\hline Blicca bjoerkna & Sapanca Lake & Soylu (2012) \\
\hline \multicolumn{3}{|l|}{$\begin{array}{l}\text { Diplozoon homoion } \\
\text { Bykhovskii and Nagibi, } 1959\end{array}$} \\
\hline Rutilus rutilus & Manyas Lake & Öztürk (2000) \\
\hline
\end{tabular}


Table 3 (continuing): Helminth - Host List.

\begin{tabular}{|c|c|c|}
\hline Alburnus alburnus & Enne Dam Lake & Koyun (2001) \\
\hline Alburnus alburnus & Mustafakemalpaşa Stream & Aydoğdu and Selver (2006) \\
\hline Cyprinus carpio & $\begin{array}{l}\text { Kepez I Hydro Electric } \\
\text { Power Plant Loading Pond }\end{array}$ & Soylu and Emre (2007) \\
\hline Pseudophoxinus antalyae & $\begin{array}{l}\text { Kepez I Hydro Electric } \\
\text { Power Plant Loading Pond }\end{array}$ & Soylu and Emre (2007) \\
\hline \multicolumn{3}{|l|}{$\begin{array}{l}\text { Diplozoon paradoxum } \\
\text { Nordman, } 1832\end{array}$} \\
\hline Rhodeus amarus & Simav, Nif Brooks & Geldiay and Balik (1974) \\
\hline Rutilus rutilus & Uluabat Lake & Geldiay and Balik (1974) \\
\hline Acantobrama marmid & Tigem Reservoirs & Zeren (2008) \\
\hline Alburnus chalcoides & Tödürge Lake & Yildirim and Ünver (2006) \\
\hline \multicolumn{3}{|l|}{$\begin{array}{l}\text { Diplozoon barbi } \\
\text { Nordman, } 1832 \\
\end{array}$} \\
\hline Capoeta trutta & Keban Dam Lake & Sağlam (1992) \\
\hline Luciobarbus pectoralis & Keban Dam Lake & Sağlam (1992) \\
\hline \multicolumn{3}{|l|}{$\begin{array}{l}\text { Dogielius forceps } \\
\text { Bychowsky, } 1936\end{array}$} \\
\hline Capoeta damascina & Murat River & Koyun (2011) \\
\hline \multicolumn{3}{|l|}{$\begin{array}{l}\text { Gyrodactylus arcuatus } \\
\text { Bychowsky, } 1933\end{array}$} \\
\hline Gasterosteus aculeatus & Sirakaraağaçlar Stream & Özer et al. (2004) \\
\hline \multicolumn{3}{|l|}{$\begin{array}{l}\text { Gyrodactylus cobitis } \\
\text { Bychowsky, } 1933\end{array}$} \\
\hline Cobitis simplicispina & Akşehir Lake & Kartal (2006) \\
\hline \multicolumn{3}{|l|}{$\begin{array}{l}\text { Gyrodactylus elegans } \\
\text { Nordmann, } 1832\end{array}$} \\
\hline Cyprinus carpio & Eski Gediz Stream & Geldiay and Balik (1974) \\
\hline Cyprinus carpio & Seyhan River & Cengizler et al. (2001) \\
\hline Luciobarbus pectoralis & Seyhan River & Şahan and Cengizler (2003) \\
\hline Barbus plebejus & Almus Dam Lake & Turgut (2005) \\
\hline Chondrostoma regium & Almus Dam Lake & Turgut (2005) \\
\hline Cyprinus carpio & Karamik Lake & Kutlu (2005) \\
\hline Cyprinus carpio & Eber Lake & Öztürk (2005) \\
\hline Cyprinus carpio & Akşehir Lake & Kartal (2006) \\
\hline Cyprinus carpio & Selevir Dam Lake & Öztürk and Bulut (2006) \\
\hline
\end{tabular}


Table 3 (continuing): Helminth - Host List.

\begin{tabular}{|l|l|l|}
\hline $\begin{array}{l}\text { Gyrodactylus gobii } \\
\text { Shul'man, 1953 }\end{array}$ & \\
\hline Neogobius fluviatilis & Uluabat Lake & Öztürk et al. (2002) \\
\hline $\begin{array}{l}\text { Gyrodactylus hemibarbi } \\
\text { (Ergens, 1980) }\end{array}$ & & \\
\hline Barbus plebejus & Almus Dam Lake & Turgut (2005) \\
\hline $\begin{array}{l}\text { Gyrodactylus katharineri } \\
\text { Malmberg, 1964 }\end{array}$ & & \\
\hline Carassius carassius & Enne Dam Lake & \\
\hline Carassius auratus & Enne Dam Lake & Koyun (2001) \\
\hline & & Koyun (2001) \\
\hline $\begin{array}{l}\text { Gyrodactylus macrocornis } \\
\text { Ergens, 1963 }\end{array}$ & & \\
\hline Chondrostoma regium & Almus Dam Lake & Turgut (2005) \\
\hline Chondrostoma regium & Almus Dam Lake & Özgül (2008) \\
\hline $\begin{array}{l}\text { Gyrodactylus medius } \\
\text { Kathariner, 1894 }\end{array}$ & & \\
\hline Tinca tinca & & \\
\hline & Kovada Lake & Kir and Özan (2005) \\
\hline $\begin{array}{l}\text { Gyrodactylus narzikulovi } \\
\text { Ergens and Dzhalilov 1979 }\end{array}$ & & \\
\hline Capoeta tinca & Almus Dam Lake & Turgut (2005) \\
\hline Capoeta tinca & Almus Dam Lake & Özgül (2008) \\
\hline $\begin{array}{l}\text { Gyrodactylus proterorhini } \\
\text { Ergens, 1967 }\end{array}$ & & \\
\hline Neogobius melanostomus & Sirakaraağaçlar Stream & Özer (2007) \\
\hline $\begin{array}{l}\text { Gyrodactylus scardinii } \\
\text { Malmberg, 1956 }\end{array}$ & & \\
\hline Cyprinus carpio & Siğirci Lake \\
\hline Carassius gibelio & Seyitler Dam Lake & \\
\hline $\begin{array}{l}\text { Onchocleidus similis } \\
\text { Mueller, 1936 }\end{array}$ & & \\
\hline Lepomis gibbosus & & \\
\hline
\end{tabular}


Table 3 (continuing): Helminth - Host List.

\begin{tabular}{|l|l|l|}
\hline $\begin{array}{l}\text { Quadriacanthus clariadis } \\
\text { (Paperna, 1961) }\end{array}$ & & \\
\hline Clarias gariepinus & $\begin{array}{l}\text { Kepez I Hydro Electric } \\
\text { Power Plant Loading Pond }\end{array}$ & Soylu and Emre (2005) \\
\hline $\begin{array}{l}\text { Paradiplozoon megan } \\
\text { Bykhovskii and Nagibi, 1959 }\end{array}$ & & \\
\hline Squalius cephalus & Doganci Dam Lake & Aydoğdu (2001) \\
\hline Squalius cephalus & Susurluk River & Gürkan and Özan (2012) \\
\hline Alburnus chalcoides & Tödürge Lake & Yildirim and Ünver (2006) \\
\hline $\begin{array}{l}\text { Tetraonchus monenteron } \\
\text { Diesing, 1858 }\end{array}$ & & \\
\hline Esox lucius & & \\
\hline Esox lucius & Sapanca Lake & Soylu (1990) \\
\hline Esox lucius & Uluabat Lake & Öztürk (1995) \\
\hline Esox lucius & Doganci Dam Lake & Aydoğdu 2001 \\
\hline Esox lucius & Gölbaşi Dam Lake & Aydoğdu et al. (2008) \\
\hline & Siğirci Lake & Çolak (2013) \\
\hline Phylum Platyhelminthes & & \\
\hline Class Digenea & & Soylu (2006) \\
\hline & & Soylu (1990) \\
\hline $\begin{array}{l}\text { Allocreadium isoporum } \\
\text { Looss, 1894 }\end{array}$ & & Soylu (1990) \\
\hline Luciobarbus escherichii & Doganci Dam Lake & Aydoğdu (2001) \\
\hline Alburnus alburnus & Enne Dam Lake & Koyun (2001) \\
\hline Barbus plebejus & Enne Dam Lake & Koyun (2001) \\
\hline Squalius cephalus & Enne Dam Lake \\
\hline Barbus plebejus & Murat River, Aras River & \\
\hline & & Aslan (2006) \\
\hline $\begin{array}{l}\text { Aspidogaster limacoides } \\
\text { Diesing, 1835 }\end{array}$ & & \\
\hline Rutilus rutilus & Sapanca Lake & \\
\hline Blicca bjoerkna & Sapanca Lake & \\
\hline Vimba vimba & Sapanca Lake & \\
\hline & & \\
\hline $\begin{array}{l}\text { Asymphylodora imitans } \\
\text { Mühling, 1898) }\end{array}$ & & \\
\hline Blicca bjoerkna & & \\
\hline & & \\
\hline & & \\
\hline & & \\
\hline
\end{tabular}


Table 3 (continuing): Helminth - Host List.

\begin{tabular}{|c|c|c|}
\hline $\begin{array}{l}\text { Asymphylodora } \\
\text { markewitschi } \\
\text { Kulakovskaya, } 1947\end{array}$ & & \\
\hline $\begin{array}{l}\text { Scardinius } \\
\text { erythrophthalmus }\end{array}$ & Uluabat Lake & Oğuz and Öztürk (1993) \\
\hline $\begin{array}{l}\text { Scardinius } \\
\text { erythrophthalmus }\end{array}$ & Kocadere Stream & Selver (2008) \\
\hline $\begin{array}{l}\text { Asymphylodora tincae } \\
\text { (Mooder, 1790) }\end{array}$ & & \\
\hline Alburnus sp. & $\begin{array}{l}\text { Gölbaşi Lake, Eymir Lake, } \\
\text { Çifteler-Sakaryabaşi Fish } \\
\text { Production Station, } \\
\text { Kurtboğazi Dam Lake, } \\
\text { Sariyar Dam Lake, } \\
\text { Kizilcahamam Brook }\end{array}$ & Burgu et al. (1988) \\
\hline Barbus sp. & $\begin{array}{l}\text { Çifteler-Sakaryabaşi Fish } \\
\text { Production Station, Sariyar } \\
\text { Dam Lake }\end{array}$ & Burgu et al. (1988) \\
\hline Esox lucius & $\begin{array}{l}\text { Gölbaşi Lake, Eymir Lake, } \\
\text { Çifteler-Sakaryabaşi Fish } \\
\text { Production Station }\end{array}$ & Burgu et al. (1988) \\
\hline Tinca tinca & Sapanca Lake & Soylu (1990) \\
\hline Tinca tinca & Uluabat Lake & Aksakal (1992) \\
\hline Tinca tinca & İznik Lake & Aydoğdu et al. (1996a) \\
\hline Tinca tinca & Terkos Lake & Soylu (2003) \\
\hline Tinca tinca & Kapulukaya Dam Lake & Yildiz (2003) \\
\hline Tinca tinca & Beyşsehir Lake & Özan (2005) \\
\hline Tinca tinca & Kovada Lake & Kir and Özan (2005) \\
\hline $\begin{array}{l}\text { Bucephalus polymorphus } \\
\text { Baer, } 1827\end{array}$ & & \\
\hline Esox lucius & Sapanca Lake & Soylu (1990) \\
\hline Silurus glanis & Sapanca Lake & Soylu (1990) \\
\hline Sander lucioperca & Egirdir Lake & Yildirim et al. (1996) \\
\hline Sander lucioperca & Beyşehir Lake, Kovada Lake & Kara (1997) \\
\hline Knipowitschia caucasica & Eğirdir Lake & Diler et al. (2001) \\
\hline $\begin{array}{l}\text { Clinostomum complanatum } \\
\text { Rudolphi, } 1819\end{array}$ & & \\
\hline
\end{tabular}


Table 3 (continuing): Helminth - Host List.

\begin{tabular}{|c|c|c|}
\hline Cyprinus carpio & $\begin{array}{l}\text { Gölbaşi Lake, Eymir Lake, } \\
\text { Çifteler-Sakaryabaşi Fish } \\
\text { Production Station, } \\
\text { Kurtboğazi Dam Lake, } \\
\text { Sariyar Dam Lake, } \\
\text { Kizilcahamam Brook, } \\
\text { Hirfanli Dam Lake, Çankiri- } \\
\text { Günerdiğin Pond }\end{array}$ & Burgu et al. (1988) \\
\hline Alburnus sp. & $\begin{array}{l}\text { Gölbaşi Lake, Eymir Lake, } \\
\text { Çifteler-Sakaryabaşi Fish } \\
\text { Production Station, } \\
\text { Kurtboğazi Dam Lake, } \\
\text { Sariyar Dam Lake, } \\
\text { Kizilcahamam Brook }\end{array}$ & Burgu et al. (1988) \\
\hline Chondrostoma sp. & $\begin{array}{l}\text { Kizilcahamam Brook, } \\
\text { Nallihan Brook }\end{array}$ & Burgu et al. (1988) \\
\hline Varicorhinus sp. & $\begin{array}{l}\text { Çifteler-Sakaryabaşi Fish } \\
\text { Production Station, } \\
\text { Kurtboğazi Dam Lake, } \\
\text { Çankiri-Günerdiğin Pond, } \\
\text { Kizilcahamam Brook, } \\
\text { Nallihan Brook }\end{array}$ & Burgu et al. (1988) \\
\hline Luciobarbus escherichii & Kirmir Stream & $\begin{array}{l}\text { Öge and Sarimehmetoğlu } \\
\text { (1996) }\end{array}$ \\
\hline Capoeta tinca & Kirmir Stream & $\begin{array}{l}\text { Öge and Sarimehmetoğlu } \\
\text { (1996) }\end{array}$ \\
\hline Perca fluviatilis & Siğirci Lake & Soylu (2013) \\
\hline $\begin{array}{l}\text { Scardinius } \\
\text { erythrophthalmus }\end{array}$ & Siğirci Lake & Çolak (2013) \\
\hline Cyprinus carpio & Siğirci Lake & Çolak (2013) \\
\hline Sander lucioperca & Siğirci Lake & Çolak (2013) \\
\hline Lepomis gibbosus & Siğirci Lake & Çolak (2013) \\
\hline \multicolumn{3}{|l|}{$\begin{array}{l}\text { Crepidostomum farionis } \\
\text { (O. F. Muller, 1780) }\end{array}$} \\
\hline Salmo trutta abanticus & Abant Lake & Caira (1989) \\
\hline \multicolumn{3}{|l|}{$\begin{array}{l}\text { Diplostomum spathaecum } \\
\text { (Rudolphi, 1819) }\end{array}$} \\
\hline Rutilus rutilus & Sapanca Lake & Soylu (1990) \\
\hline $\begin{array}{l}\text { Scardinius } \\
\text { erythrophthalmus }\end{array}$ & Sapanca Lake & Soylu (1990) \\
\hline Blicca bjoerkna & Sapanca Lake & Soylu (1990) \\
\hline Esox lucius & Sapanca Lake & Soylu (1990) \\
\hline Silurus glanis & Sapanca Lake & Soylu (1990) \\
\hline
\end{tabular}


Table 3 (continuing): Helminth - Host List.

\begin{tabular}{|c|c|c|}
\hline Tinca tinca & Sapanca Lake & Soylu (1990) \\
\hline Tinca tinca & Terkos Lake & Soylu (2003) \\
\hline Abramis brama & Terkos Lake & Karatoy (2004) \\
\hline $\begin{array}{l}\text { Scardinius } \\
\text { erythrophthalmus }\end{array}$ & Terkos Lake & Kahveci (2004) \\
\hline Esox lucius & Gölbaşi Dam Lake & Aydoğdu et al. (2008) \\
\hline Blicca bjoerkna & Kocadere Stream & Selver (2008) \\
\hline Rutilus rutilus & Kocadere Stream & Selver (2008) \\
\hline $\begin{array}{l}\text { Scardinius } \\
\text { erythrophthalmus }\end{array}$ & Kocadere Stream & Selver (2008) \\
\hline \multicolumn{3}{|l|}{$\begin{array}{l}\text { Diplodiscus subclavatus } \\
\text { Diesing, } 1836\end{array}$} \\
\hline Esox lucius & Uluabat Lake & Öztürk et al. (2000) \\
\hline \multicolumn{3}{|l|}{$\begin{array}{l}\text { Opisthorchis felinus } \\
\text { (Rivolta, 1884) }\end{array}$} \\
\hline Rutilus rutilus & Uluabat, Manyas Lakes & Geldiay and Balik (1974) \\
\hline \multicolumn{3}{|l|}{$\begin{array}{l}\text { Orientocreadium siluri } \\
\text { Bykhovski and Dubi., } 1954\end{array}$} \\
\hline Silurus glanis & Sapanca Lake & Soylu (1990) \\
\hline \multicolumn{3}{|l|}{$\begin{array}{l}\text { Orientocreadium } \\
\text { batrachoides } \\
\text { Tubangui, } 1931\end{array}$} \\
\hline Clarias gariepinus & Asi River & Tepe et al. (2013) \\
\hline \multicolumn{3}{|l|}{$\begin{array}{l}\text { Phyllodistomum elongatum } \\
\text { Nybelin, } 1926\end{array}$} \\
\hline Luciobarbus pectoralis & Karacaören I Dam Lake & Kir (1998) \\
\hline \multicolumn{3}{|l|}{$\begin{array}{l}\text { Posthodiplostomum cuticola } \\
\text { Nordmann, } 1832\end{array}$} \\
\hline Scardinius erythrophthalmus & Sapanca Lake & Soylu (1990) \\
\hline Blicca bjoerkna & Sapanca Lake & Soylu (1990) \\
\hline Blicca bjoerkna & Uluabat Lake & Akinci (1999) \\
\hline Cyprinus carpio & Eber Lake & Öztürk (2005) \\
\hline Vimba vimba & Sapanca Lake & Uzunay (2006) \\
\hline Alburnus chalcoides & Tödürge Lake & Yildirim and Ünver (2006) \\
\hline Scardinius erythropthalmus & Siğirci Lake & Çolak (2013) \\
\hline \multicolumn{3}{|l|}{$\begin{array}{l}\text { Posthodiplostomum } \\
\text { minimum Maccallum, } 1921\end{array}$} \\
\hline Salmo trutta fario & Munzur Stream & Ekingen (1976) \\
\hline
\end{tabular}


Table 3 (continuing): Helminth - Host List.

\begin{tabular}{|c|c|c|}
\hline \multicolumn{3}{|l|}{$\begin{array}{l}\text { Pygidiopsis genata } \\
\text { Looss, } 1907\end{array}$} \\
\hline Neogobius melanostomus & $\begin{array}{l}\text { Sirakaraağaçlar } \\
\text { Stream }\end{array}$ & Özer (2007) \\
\hline \multicolumn{3}{|l|}{$\begin{array}{l}\text { Rhipidocotyle fennica } \\
\text { Taskinen, } 1991\end{array}$} \\
\hline Esox lucius & Uluabat Lake & Öztürk et al. (2000) \\
\hline \multicolumn{3}{|l|}{$\begin{array}{l}\text { Tylodelphys clavata } \\
\text { Nordmann, } 1832\end{array}$} \\
\hline Rutilus rutilus & Sapanca Lake & Soylu (1990) \\
\hline $\begin{array}{l}\text { Scardinius } \\
\text { erythrophthalmus }\end{array}$ & Sapanca Lake & Soylu (1990) \\
\hline Blicca bjoerkna & Sapanca Lake & Soylu (1990) \\
\hline Esox lucius & Sapanca Lake & Soylu (1990) \\
\hline Silurus glanis & Sapanca Lake & Soylu (1990) \\
\hline Tinca tinca & Sapanca Lake & Soylu (1990) \\
\hline Tinca tinca & Terkos Lake & Soylu (2003) \\
\hline Abramis brama & Terkos Lake & Karatoy (2004) \\
\hline $\begin{array}{l}\text { Scardinius } \\
\text { erythrophthalmus }\end{array}$ & Terkos Lake & Kahveci (2004) \\
\hline Vimba vimba & Sapanca Lake & Uzunay (2006) \\
\hline Perca fluviatilis & Siğirci Lake & Soylu (2013) \\
\hline Atherina boyeri & İznik Lake & Çolak (2013) \\
\hline Scardinius erytrophthalmus & Siğirci Lake & Çolak (2013) \\
\hline Sander lucioperca & Siğirci Lake & Çolak (2013) \\
\hline Cyprinus carpio & Siğirci Lake & Çolak (2013) \\
\hline Lepomis gibbosus & Siğirci Lake & Çolak (2013) \\
\hline Rutilus rutilus & Siğirci Lake & Çolak (2013) \\
\hline Esox lucius & Siğirci Lake & Çolak (2013) \\
\hline Silurus glanis & Siğgirci Lake & Çolak (2013) \\
\hline \multicolumn{3}{|l|}{$\begin{array}{l}\text { Tetracotyle percae- } \\
\text { fluviatilis (Linstow, 1856) }\end{array}$} \\
\hline Abramis brama & Terkos Lake & Karatoy (2004) \\
\hline $\begin{array}{l}\text { Scardinius } \\
\text { erythrophthalmus }\end{array}$ & Terkos Lake & Kahveci (2004) \\
\hline
\end{tabular}


Table 3 (continuing): Helminth - Host List.

\begin{tabular}{|c|c|c|}
\hline \multicolumn{3}{|l|}{ Phylum Platyhelminthes } \\
\hline Class Cestoda & & \\
\hline $\begin{array}{l}\text { Bathybothrium rectangulum } \\
\text { (Bloch, 1782) }\end{array}$ & & \\
\hline Esox lucius & Işikli Dam Lake & Dişçi (2002) \\
\hline $\begin{array}{l}\text { Biacetabulum } \\
\text { appendiculatum } \\
\text { Szidat, } 1937\end{array}$ & & \\
\hline Luciobarbus escherichii & Enne Dam Lake & Koyun (2001) \\
\hline $\begin{array}{l}\text { Bothriocephalus } \\
\text { acheilognathi } \\
\text { Yamaguti, } 1955\end{array}$ & & \\
\hline Cyprinus carpio & İznik Lake & Türkmen (1990) \\
\hline Rutilus frisii & İznik Lake & Türkmen (1990) \\
\hline Cyprinus carpio & $\begin{array}{l}\text { Karasu Brook, Bendimahi } \\
\text { Brook, Engil Brook, Zernek } \\
\text { Dam Lake }\end{array}$ & Topçu (1993) \\
\hline Capoeta umbla & Hazar Lake & Aksoy (1996) \\
\hline Tinca tinca & Mogan Lake & Erkul (1997) \\
\hline Cyprinus carpio & $\begin{array}{l}\text { Mogan Lake, Hirfanli Dam } \\
\text { Lake, Kirmir Brook }\end{array}$ & Erkul (1997) \\
\hline Cyprinus carpio & Karacaören I Dam Lake & Kir (1998) \\
\hline Cyprinus carpio & Manyas Lake & Öztürk (2000) \\
\hline Alburnus mossulensis & Manyas Lake & Öztürk (2000) \\
\hline Alburnus alburnus & Enne Dam Lake & Koyun (2001) \\
\hline Luciobarbus escherichii & Doganci Dam Lake & Aydoğdu (2001) \\
\hline Squalius cephalus & Doganci Dam Lake & Aydoğdu (2001) \\
\hline Neogobius fluviatilis & Uluabat Lake & Öztürk et al. (2002) \\
\hline Silurus glanis & Hirfanli Dam Lake & Aydin (2003) \\
\hline Capoeta umbla & Karakaya Dam Lake & Örün et al. (2003) \\
\hline Chondrostoma regium & Karakaya Dam Lake & Örün et al. (2003) \\
\hline Cyprinus carpio & Karakaya Dam Lake & Örün et al. (2003) \\
\hline Acanthobrama marmid & Karakaya Dam Lake & Örün et al. (2003) \\
\hline Alburnus mossulensis & Karakaya Dam Lake & Örün et al. (2003) \\
\hline Squalius cephalus & Karakaya Dam Lake & Örün et al. (2003) \\
\hline Cyprinus carpio & Karamik Lake & Kutlu (2005) \\
\hline Tinca tinca & Beyşehir Lake & Özan (2005) \\
\hline Cyprinus carpio & Beyşehir Lake & Özan (2005) \\
\hline Cyprinus carpio & Eber Lake & Öztürk (2005) \\
\hline Tinca tinca & Kovada Lake & Kir and Özan (2005) \\
\hline
\end{tabular}


Table 3 (continuing): Helminth - Host List.

\begin{tabular}{|c|c|c|}
\hline Capoeta trutta & Keban Dam Lake & Dörücü and İspir (2005) \\
\hline Capoeta umbla & Keban Dam Lake & Dörücü and İspir (2005) \\
\hline Chondrostoma regium & Keban Dam Lake & Dörücü and İspir (2005) \\
\hline Cyprinus carpio & Keban Dam Lake & Dörücü and İspir (2005) \\
\hline Tinca tinca & Sapanca Lake & Akbeniz (2006) \\
\hline Cyprinus carpio & Sapanca Lake & Uzunay (2006) \\
\hline Blicca bjoerkna & Sapanca Lake & Soylu (2006) \\
\hline Alburnus alburnus & Mustafakemalpaşa Stream & Aydoğdu and Selver (2006) \\
\hline Cyprinus carpio & Selevir Dam Lake & Öztürk and Bulut (2006) \\
\hline Cyprinus carpio & Kovada Lake & Kir and Özan (2007) \\
\hline Rutilus rutilus & Kocadere Stream & Selver (2008) \\
\hline Cyprinus carpio & Murat River, Aras River & Aslan (2009) \\
\hline Squalius cephalus & Örenler Dam Lake & Kurupinar (2009) \\
\hline Cyprinus carpio & Karacaören II Dam Lake & Samanci (2011) \\
\hline Alburnus chalcoides & Tödürge Lake & Yildirim and Ünver (2006) \\
\hline Atherina boyeri & İznik Lake & Çolak (2013) \\
\hline Cyprinus carpio & Siğirci Lake & Çolak (2013) \\
\hline \multicolumn{3}{|l|}{$\begin{array}{l}\text { Caryophyllaeides fennica } \\
\text { (Schneider, 1902) }\end{array}$} \\
\hline Rutilus rutilus & Manyas Lake & Öztürk (2000) \\
\hline Scardinius erythrophthalmus & Manyas Lake & Öztürk (2000) \\
\hline \multicolumn{3}{|l|}{$\begin{array}{l}\text { Caryophyllaeus brachycollis } \\
\text { Janiszewska, } 1951\end{array}$} \\
\hline Cyprinus carpio & Antakya Lake & Paperna (1964) \\
\hline \multicolumn{3}{|l|}{$\begin{array}{l}\text { Caryophyllaeus fimbriceps } \\
\text { Annenkova-Khlopina, } 1919\end{array}$} \\
\hline Cyprinus carpio & Siğirci Lake & Çolak (2013) \\
\hline \multicolumn{3}{|l|}{$\begin{array}{l}\text { Caryophyllaeus laticeps } \\
\text { (Müller, 1781) }\end{array}$} \\
\hline Cyprinus carpio & $\begin{array}{l}\text { Gölbaşi Lake, Eymir Lake, } \\
\text { Çifteler-Sakaryabaşi Fish } \\
\text { Production Station, } \\
\text { Kurtboğazi Dam Lake, } \\
\text { Sariyar Dam Lake, } \\
\text { Kizilcahamam Brook, } \\
\text { Hirfanli Dam Lake, Çankiri- } \\
\text { Günerdiğin Pond }\end{array}$ & Burgu et al. (1988) \\
\hline
\end{tabular}


Table 3 (continuing): Helminth - Host List.

\begin{tabular}{|c|c|c|}
\hline Cyprinus carpio & İznik Lake & Türkmen (1990) \\
\hline Cyprinus carpio & $\begin{array}{l}\text { Karasu Brook, Bendimahi } \\
\text { Brook, Engil Brook, Zernek } \\
\text { Dam Lake }\end{array}$ & Topçu (1993) \\
\hline Rutilus frisii & İznik Lake & Aydoğdu et al. (1997b) \\
\hline Capoeta umbla & Hazar Lake & Aksoy (1996) \\
\hline Cyprinus carpio & Karacaören I Dam Lake & Kir (1998) \\
\hline Cyprinus carpio & Kovada Lake & Becer and Kara (1998) \\
\hline Blicca bjoerkna & Manyas Lake & Öztürk (2000) \\
\hline Vimba vimba & Manyas Lake & Öztürk (2000) \\
\hline Cyprinus carpio & Manyas Lake & Öztürk (2000) \\
\hline Cyprinus carpio & Karacabey Lagoon Lake & Aydoğdu et al. (2001a) \\
\hline Luciobarbus escherichii & Doğanci Dam Lake & Aydoğdu (2001) \\
\hline Cyprinus carpio & Seyhan River & Şahan and Cengizler (2003) \\
\hline Luciobarbus pectoralis & Seyhan River & Şahan and Cengizler (2003) \\
\hline Abramis brama & Terkos Lake & Karatoy (2004) \\
\hline Cyprinus carpio & Beyşehir Lake & Özan (2005) \\
\hline Tinca tinca & Beyşehir Lake & Özan (2005) \\
\hline Tinca tinca & Kovada Lake & Kir and Özan (2005) \\
\hline Tinca tinca & Sapanca Lake & Akbeniz (2006) \\
\hline Cyprinus carpio & Sapanca Lake & Uzunay (2006) \\
\hline Blicca bjoerkna & Sapanca Lake & Soylu (2006) \\
\hline Cyprinus carpio & Selevir Dam Lake & Öztürk and Bulut (2006) \\
\hline Cyprinus carpio & $\begin{array}{l}\text { Kepez I Hydro Electric } \\
\text { Power Plant Loading Pond }\end{array}$ & Soylu and Emre (2007) \\
\hline Vimba vimba & Gölbaşi Dam Lake & Aydoğdu et al. (2008) \\
\hline Blicca bjoerkna & Kocadere Stream & Selver (2008) \\
\hline Capoeta capoeta & Murat River, Aras River & Aslan (2009) \\
\hline Acanthobrama marmid & Murat River, Aras River & Aslan (2009) \\
\hline Cyprinus carpio & Karacaören II Dam Lake & Samanci (2011) \\
\hline \multicolumn{3}{|l|}{$\begin{array}{l}\text { Khawia armeniaca } \\
\text { Cholodkovskii, } 1878\end{array}$} \\
\hline Luciobarbus pectoralis & Keban Dam Lake & $\begin{array}{l}\text { Özdemir and Sarieyyüboğlu } \\
\text { (1993) }\end{array}$ \\
\hline Capoeta umbla & Hazar Lake & Aksoy (1996) \\
\hline Acanthobrama marmid & Karakaya Dam Lake & Örün et al. (2003) \\
\hline Squalius cephalus & Karakaya Dam Lake & Örün et al. (2003) \\
\hline Capoeta trutta & Karakaya Dam Lake & Örün et al. (2003) \\
\hline Cyprinus carpio & Karakaya Dam Lake & Örün et al. (2003) \\
\hline Capoeta umbla & Karakaya Dam Lake & Örün et al. (2003) \\
\hline Capoeta umbla & Keban Dam Lake & Dörücü and İspir (2005) \\
\hline Cyprinus carpio & Keban Dam Lake & Dörücü and İspir (2005) \\
\hline
\end{tabular}


Table 3 (continuing): Helminth - Host List.

\begin{tabular}{|c|c|c|}
\hline $\begin{array}{l}\text { Ligula intestinalis } \\
\text { Lin., } 1758\end{array}$ & & \\
\hline Capoeta umbla & Cip Lake & Cantoray and Özcan (1975) \\
\hline Squalius cephalus & Cip Lake & Cantoray and Özcan (1975) \\
\hline Luciobarbus escherichii & Cip Lake & Cantoray and Özcan (1975) \\
\hline Acanthobrama marmid & Devegecidi Dam Lake & Başaran and Kelle (1976) \\
\hline Alburnus mossulensis & Devegecidi Dam Lake & Başaran and Kelle (1976) \\
\hline Acanthobrama marmid & Devegecidi Dam Lake & Kelle (1978) \\
\hline Alburnus mossulensis & Devegecidi Dam Lake & Kelle (1978) \\
\hline Silurus glanis & Sariyar Dam Lake & Keskin and Erakan (1987) \\
\hline Chondrostoma regium & Keban Dam Lake & Keskin and Erakan (1987) \\
\hline Vimba vimba & Kumkaya Dam Lake & Keskin and Erakan (1987) \\
\hline Capoeta capoeta & Demirkopru Dam Lake & Keskin and Erakan (1987) \\
\hline Alburnus orontis & $\begin{array}{l}\text { Kizilirmak Brook, Yeniköy } \\
\text { Stream, Çayirhan Stream, } \\
\text { Kumkaya Dam Lake, Enne } \\
\text { Dam Lake }\end{array}$ & Keskin and Erakan (1987) \\
\hline Garra rufa & Cag-cag Stream & Keskin and Erakan (1987) \\
\hline Squalius cephalus & $\begin{array}{l}\text { Hamidiye, Pazarkavşaği } \\
\text { Bridge }\end{array}$ & Keskin and Erakan (1987) \\
\hline Alburnus sp. & $\begin{array}{l}\text { Gölbaşi Lake, Eymir Lake, } \\
\text { Çifteler-Sakaryabaşi Fish } \\
\text { Production Station, } \\
\text { Kurtboğazi Dam Lake, } \\
\text { Sariyar Dam Lake, } \\
\text { Kizilcahamam Brook }\end{array}$ & Burgu et al. (1988) \\
\hline Esox lucius & $\begin{array}{l}\text { Gölbaşi Lake, Eymir Lake, } \\
\text { Çifteler-Sakaryabaşi Fish } \\
\text { Production Station }\end{array}$ & Burgu et al. (1988) \\
\hline Tinca tinca & Mogan Lake & Burgu et al. (1988) \\
\hline Vimba vimba & Sariyar Dam Lake & Ekmekçi (1989) \\
\hline Alburnus orontis & Almus Dam Lake & Cengizler et al. (1991) \\
\hline Squalius cephalus & Almus Dam Lake & Cengizler et al. (1991) \\
\hline Luciobarbus pectoralis & Keban Dam Lake & $\begin{array}{l}\text { Özdemir and Sarieyyüboğlu } \\
\text { (1993) }\end{array}$ \\
\hline Alburnus alburnus & Upper Porsuk Basin & Yilmaz et al. (1996) \\
\hline Cyprinus carpio & Kovada Lake & Becer and Kara (1998) \\
\hline Cyprinus carpio & Karacaören I Dam Lake & Kir (1998) \\
\hline Luciobarbus pectoralis & Karacaören I Dam Lake & Kir (1998) \\
\hline Alburnus alburnus & Kutahya Region Lakes & Koyun et al. (1999) \\
\hline Squalius cephalus & Yeşilköy Pond & Bulgen (1999) \\
\hline Alburnus mossulensis & Manyas Lake & Öztürk (2000) \\
\hline Rutilus rutilus & Manyas Lake & Öztürk (2000) \\
\hline
\end{tabular}


Table 3 (continuing): Helminth - Host List.

\begin{tabular}{|c|c|c|}
\hline Acanthobrama marmid & Keban Dam Lake & Türk (2000) \\
\hline Alburnus alburnus & Enne Dam Lake & Koyun (2001) \\
\hline Tinca tinca & Beyşehir Lake & Yildiz et al. (2003) \\
\hline Rutilus rutilus & Yenice Pond & Oğuz et al. (2004) \\
\hline Alburnus orontis & Camkoru Pond & İnnal (2004) \\
\hline Scardinius erythrophthalmus & Terkos Lake & Kahveci (2004) \\
\hline Squalius cephalus & Gelingüllü Dam Lake & $\begin{array}{l}\text { Ekmekçi and Kirankaya } \\
\text { (2004) }\end{array}$ \\
\hline Tinca tinca & Kovada Lake & Kir and Özan (2005) \\
\hline Capoeta bergamae & Topcam Dam Lake & Şaşi (2005) \\
\hline Squalius cephalus & Caparlipatlak Dam Lake & Torcu-Koç et al. (2006) \\
\hline Chondrostoma regium & Almus Dam Lake & Özgül and Turgut (2006) \\
\hline Rhodeus amarus & Sapanca Lake & Akmirza (2007) \\
\hline Alburnus orontis & Camlidere Dam Lake & İnnal et al. (2007) \\
\hline Alburnus orontis & Kirmir Creek & İnnal et al. (2007) \\
\hline Alburnus orontis & Bulak Stream & İnnal et al. (2007) \\
\hline Alburnus orontis & Mogan Lake & İnnal et al. (2007) \\
\hline Alburnoides bipunctatus & Kirmir Creek & İnnal et al. (2007) \\
\hline Cyprinus carpio & Camlidere Dam Lake & İnnal et al. (2007) \\
\hline Cyprinus carpio & Aksu River & İnnal et al. (2007) \\
\hline Tinca tinca & Abant Lake & İnnal et al. (2007) \\
\hline Tinca tinca & Yeniçağa Lake & İnnal et al. (2007) \\
\hline Vimba vimba & Yamansaz Lake & İnnal et al. (2007) \\
\hline Barbus plebejus & Çildir Lake & İnnal et al. (2007) \\
\hline Barbus plebejus & Barhal Creek & İnnal et al. (2007) \\
\hline Barbus plebejus & Almus Dam Lake & Develi (2008) \\
\hline Chondrostoma nasus & Kunduzlar Dam Lake & Özbek (2009) \\
\hline Squalius cephalus & Kunduzlar Dam Lake & Özbek (2009) \\
\hline Alburnus escherichii & Kunduzlar Dam Lake & Özbek (2009) \\
\hline Squalius cephalus & Örenler Dam Lake & Kurupinar (2009) \\
\hline Alburnus escherichii & Çamkoru Pond & İnnal et al. (2010) \\
\hline Gobio gobio & Çamkoru Pond & İnnal et al. (2010) \\
\hline Squalius cephalus & Çamkoru Pond & İnnal et al. (2010) \\
\hline Alburnus orontis & Almus Dam Lake & Turgut et al. (2011) \\
\hline Squalius cephalus & Almus Dam Lake & Turgut et al. (2011) \\
\hline Cyprinus carpio & Almus Dam Lake & Turgut et al. (2011) \\
\hline Capoeta tinca & Almus Dam Lake & Turgut et al. (2011) \\
\hline Capoeta capoeta & Almus Dam Lake & Turgut et al. (2011) \\
\hline \multicolumn{3}{|l|}{$\begin{array}{l}\text { Ligula pavlovskii } \\
\text { Dubinina, } 1959\end{array}$} \\
\hline Neogobius fluviatilis & Manyas Lake & Öztürk (2000) \\
\hline Neogobius fluviatilis & Uluabat Lake & Öztürk et al. (2002) \\
\hline
\end{tabular}


Table 3 (continuing): Helminth - Host List.

\begin{tabular}{|c|c|c|}
\hline $\begin{array}{l}\text { Monobothrium auriculatum } \\
\text { Kulakovskaya, } 1961\end{array}$ & & \\
\hline Capoeta damascina & Hazar Lake & Aksoy (1996) \\
\hline $\begin{array}{l}\text { Paradilepis scolecina } \\
\text { Rudolphi, } 1935\end{array}$ & & \\
\hline Cyrprinus carpio & Akşehir Lake & Buhurcu (2006) \\
\hline $\begin{array}{l}\text { Proteocephalus osculatus } \\
\text { (Goeze, 1782) }\end{array}$ & & \\
\hline Silurus glanis & $\begin{array}{l}\text { Gölbaşi Lake, Eymir Lake, } \\
\text { Sariyar Dam Lake }\end{array}$ & Burgu et al. (1988) \\
\hline Silurus glanis & Hirfanli Dam Lake & Aydin (2003) \\
\hline $\begin{array}{l}\text { Proteocephalus percae } \\
\text { (Müler, 1780) }\end{array}$ & & \\
\hline Perca fluviatilis & Siğirci Lake & Soylu (2013) \\
\hline Sander lucioperca & Siğirci Lake & Soylu (2013) \\
\hline $\begin{array}{l}\text { Proteocephalus torulosus } \\
\text { (Batsch, 1786) }\end{array}$ & & \\
\hline Tinca tinca & Kovada Lake & Kir and Özan (2005) \\
\hline Tinca tinca & Beyşehir Lake & Özan (2005) \\
\hline $\begin{array}{l}\text { Polyonchobothrium } \\
\text { magnum (Zme'ev, 1936) }\end{array}$ & & \\
\hline Clarias gariepinus & $\begin{array}{l}\text { Kepez I Hydro Electric } \\
\text { Power Plant Loading Pond }\end{array}$ & Soylu and Emre (2005) \\
\hline $\begin{array}{l}\text { Postgangesia inarmata } \\
\text { de Chambrier, Al-Kallak } \\
\text { and Mariaux, } 2003\end{array}$ & & \\
\hline Silurus triostegus & Atatürk Dam Lake & Öktener and Alaş (2009) \\
\hline $\begin{array}{l}\text { Senga mastacembeli } \\
\text { Rahemo, } 1996\end{array}$ & & \\
\hline $\begin{array}{l}\text { Mastacembelus } \\
\text { mastacembelus }\end{array}$ & Atatürk Dam Lake & Öktener and Alaş (2009) \\
\hline $\begin{array}{l}\text { Silurotaenia siluri } \\
\text { (Batsch, 1786) }\end{array}$ & & \\
\hline Silurus glanis & Sapanca Lake & Soylu (1990) \\
\hline
\end{tabular}


Table 3 (continuing): Helminth - Host List.

\begin{tabular}{|c|c|c|}
\hline $\begin{array}{l}\text { Triaenophorus crassus } \\
\text { Forel, } 1880\end{array}$ & & \\
\hline Esox lucius & Sapanca Lake & Soylu (1990) \\
\hline Phylum Nematoda & & \\
\hline $\begin{array}{l}\text { Camallanus truncatus } \\
\text { (Rudolphi, 1814) }\end{array}$ & & \\
\hline Esox lucius & Işikli Dam Lake & Dişçi (2002) \\
\hline $\begin{array}{l}\text { Eustrongylides excisus } \\
\text { Jagerskiold, } 1909\end{array}$ & & \\
\hline Neogobius fluviatilis & Manyas Lake & Öztürk (2000) \\
\hline Neogobius fluviatilis & Uluabat Lake & Öztürk et al. (2002) \\
\hline Abramis brama & Terkos Lake & Karatoy (2004) \\
\hline Scardinius erythrophthalmus & Terkos Lake & Kahveci (2004) \\
\hline Silurus glanis & Terkos Lake & Soylu (2005) \\
\hline Aphanius mento & Kirkgöz Stream & Aydoğdu et al. (2011) \\
\hline Pseudophoxinus battalgilae & Manavgat River & Aydoğdu et al. (2011) \\
\hline Perca fluviatilis & Siğirci Lake & Soylu (2013) \\
\hline Atherina boyeri & İznik Lake & Çolak (2013) \\
\hline Sander lucioperca & Siğirci Lake & Çolak (2013) \\
\hline $\begin{array}{l}\text { Molnaria intestinalis } \\
\text { Dogiel and Bychowsky, } 1934\end{array}$ & & \\
\hline Squalius lepidus & Atatürk Dam Lake & Dal (2006) \\
\hline $\begin{array}{l}\text { Philometra intestinalis } \\
\text { Dogiel and Bykhovs., } 1934\end{array}$ & & \\
\hline Chondrostoma sp. & $\begin{array}{l}\text { Kizilcahamam Brook, } \\
\text { Nallihan Brook }\end{array}$ & Burgu et al. (1988) \\
\hline $\begin{array}{l}\text { Philometra ovate } \\
\text { (Zeder, 1803) }\end{array}$ & & \\
\hline Squalius cephalus & Seydisuyu Stream & Keskin (1988) \\
\hline Capoeta umbla & Hazar Lake & Aksoy (1996) \\
\hline Squalius cephalus & Enne Dam Lake & Koyun (2001) \\
\hline Squalius cephalus & Çamkoru Lake & İnnal and Keskin (2005) \\
\hline $\begin{array}{l}\text { Philometra rischta } \\
\text { Skrjabin, } 1923\end{array}$ & & \\
\hline Capoeta umbla & Hazar Lake & Aksoy et al. (2006) \\
\hline
\end{tabular}


Table 3 (continuing): Helminth - Host List.

\begin{tabular}{|c|c|c|}
\hline \multicolumn{3}{|l|}{$\begin{array}{l}\text { Pseudocapillaria tomentosa } \\
\text { (Dujardin, 1843) }\end{array}$} \\
\hline Cyprinus carpio & Manyas Lake & Öztürk (2000) \\
\hline \multicolumn{3}{|l|}{$\begin{array}{l}\text { Raphidascaris acus } \\
\text { (Bloch, 1779) }\end{array}$} \\
\hline Esox lucius & Sapanca Lake & Soylu (1990) \\
\hline Esox lucius & Uluabat Lake & Öztürk (1995) \\
\hline Esox lucius & Karacabey Lagoon Lake & Öztürk et al. (2002) \\
\hline Esox lucius & Işikli Dam Lake & Dişçi (2002) \\
\hline Esox lucius & Gölbaşi Dam Lake & Aydoğdu et al. (2008) \\
\hline \multicolumn{3}{|l|}{$\begin{array}{l}\text { Rhabdochona denudata } \\
\text { (Dujardin, 1845) }\end{array}$} \\
\hline Cyprinus carpio & $\begin{array}{l}\text { Karasu Brook, Bendimahi } \\
\text { Brook, Engil Brook, Zernek } \\
\text { Dam Lake }\end{array}$ & Topçu (1993) \\
\hline Luciobarbus pectoralis & Karacaören I Dam Lake & Kir (1998) \\
\hline Squalius cephalus & Doganci Dam Lake & Aydoğdu (2001) \\
\hline Alburnus alburnus & Mustafakemalpaşa Stream & Aydoğdu and Selver (2006) \\
\hline Capoeta capoeta & Murat River, Aras River & Aslan (2009) \\
\hline Barbus plebejus & Murat River, Aras River & Aslan (2009) \\
\hline Barbus musra & Murat River, Aras River & Aslan (2009) \\
\hline Capoeta antalyensis & Köprüçay River & Aydoğdu et al. (2011) \\
\hline \multicolumn{3}{|l|}{$\begin{array}{l}\text { Skrjabillanus scardinii } \\
\text { Molnár, } 1966\end{array}$} \\
\hline $\begin{array}{l}\text { Scardinius } \\
\text { erythrophthalmus }\end{array}$ & Kocadere Stream & Selver (2008) \\
\hline \multicolumn{3}{|l|}{$\begin{array}{l}\text { Spiroxys contortus } \\
\text { Rudolphi, } 1819\end{array}$} \\
\hline Aphanius chantrei & Sarikum Lagoon Lake & Öztürk (2005) \\
\hline Aphanius danfordii & Sarikum Lagoon Lake & Öztürk and Özer (2008a) \\
\hline \multicolumn{3}{|l|}{ Phylum Acanthocephala } \\
\hline \multicolumn{3}{|l|}{$\begin{array}{l}\text { Acanthocephalus anguillae } \\
\text { Müller, } 1780\end{array}$} \\
\hline Esox lucius & Uluabat Lake & Öztürk (1995) \\
\hline Tinca tinca & Beyşehir Lake & Özan (2005) \\
\hline
\end{tabular}


Table 3 (continuing): Helminth - Host List.

\begin{tabular}{|c|c|c|}
\hline $\begin{array}{l}\text { Neoechinorhynchus rutili } \\
\text { (Müller, 1780) }\end{array}$ & & \\
\hline Alburnus sp. & $\begin{array}{l}\text { Gölbaşi Lake, Eymir Lake, } \\
\text { Çifteler-Sakaryabaşi Fish } \\
\text { Production Station, } \\
\text { Kurtboğazi Dam Lake, } \\
\text { Sariyar Dam Lake, } \\
\text { Kizilcahamam Brook }\end{array}$ & Burgu et al. (1988) \\
\hline Barbus sp. & $\begin{array}{l}\text { Çifteler-Sakaryabaşi Fish } \\
\text { Production Station, Sariyar } \\
\text { Dam Lake }\end{array}$ & Burgu et al. (1988) \\
\hline Cyprinus carpio & İznik Lake & Türkmen (1990) \\
\hline Esox lucius & Sapanca Lake & Soylu (1990) \\
\hline Cyprinus carpio & $\begin{array}{l}\text { Karasu Brook, Bendimahi } \\
\text { Brook, Engil Brook, Zernek } \\
\text { Dam Lake }\end{array}$ & Topçu (1993) \\
\hline Cyprinus carpio & İznik Lake & Aydoğdu et al. (1997a) \\
\hline Rutilus frisii & İznik Lake & Aydoğdu et al. (1997b) \\
\hline Rutilus rubilio & İznik Lake & Aydoğdu et al. (2000) \\
\hline Esox lucius & Işikli Dam Lake & Dişçi (2002) \\
\hline Capoeta trutta & Keban Dam Lake & $\begin{array}{l}\text { Sarieyyüboğlu and Sağlam } \\
\text { (2002) }\end{array}$ \\
\hline Capoeta trutta & Karakaya Dam Lake & Örün et al. (2003) \\
\hline Capoeta umbla & Karakaya Dam Lake & Örün et al. (2003) \\
\hline Chondrostoma regium & Karakaya Dam Lake & Örün et al. (2003) \\
\hline Cyprinus carpio & Karakaya Dam Lake & Örün et al. (2003) \\
\hline Luciobarbus esocinus & Karakaya Dam Lake & Örün et al. (2003) \\
\hline Luciobarbus esocinus & Keban Dam Lake & Dörücü and İspir (2005) \\
\hline Capoeta umbla & Keban Dam Lake & Dörücü and İspir (2005) \\
\hline Chondrostoma regium & Keban Dam Lake & Dörücü and İspir (2005) \\
\hline Cyprinus carpio & Keban Dam Lake & Dörücü and İspir (2005) \\
\hline Aphanius chantrei & Sarikum Lagoon Lake & Öztürk (2005) \\
\hline Vimba vimba & Sapanca Lake & Uzunay (2006) \\
\hline Capoeta trutta & Atatürk Dam Lake & Dal (2006) \\
\hline Neogobius melanostomus & Sirakaraağaçlar Stream & Özer (2007) \\
\hline Aphanius danfordii & Sarikum Lagoon Lake & Öztürk and Özer (2008a) \\
\hline $\begin{array}{l}\text { Neoechinorhynchus } \\
\text { zabensis Amin, Abdullah, } \\
\text { and Mhaisen, } 2003\end{array}$ & & \\
\hline Capoeta barroisi & Murat River & Oğuz et al. (2012) \\
\hline $\begin{array}{l}\text { Pomphorhynchus laevis } \\
\text { (Müller, 1776) }\end{array}$ & & \\
\hline
\end{tabular}


Table 3 (continuing): Helminth - Host List.

\begin{tabular}{|c|c|c|}
\hline Cyprinus carpio & $\begin{array}{l}\text { Gölbaşi Lake, Eymir Lake, } \\
\text { Çifteler-Sakaryabaşi Fish } \\
\text { Production Station, } \\
\text { Kurtboğazi Dam Lake, } \\
\text { Sariyar Dam Lake, } \\
\text { Kizilcahamam Brook, } \\
\text { Hirfanli Dam Lake, Çankiri- } \\
\text { Günerdiğin Pond }\end{array}$ & Burgu et al. (1988) \\
\hline Alburnus sp. & $\begin{array}{l}\text { Gölbaşi Lake, Eymir Lake, } \\
\text { Çifteler-Sakaryabaşi Fish } \\
\text { Production Station, } \\
\text { Kurtboğazi Dam Lake, } \\
\text { Sariyar Dam Lake, } \\
\text { Kizilcahamam Brook }\end{array}$ & Burgu et al. (1988) \\
\hline Silurus glanis & $\begin{array}{l}\text { Gölbaşi Lake, Eymir Lake, } \\
\text { Sariyar Dam Lake }\end{array}$ & Burgu et al. (1988) \\
\hline Esox lucius & $\begin{array}{l}\text { Gölbaşi Lake, Eymir Lake, } \\
\text { Çifteler-Sakaryabaşi Fish } \\
\text { Production Station }\end{array}$ & Burgu et al. (1988) \\
\hline Luciobarbus escherichii & $\begin{array}{l}\text { Karasu (Sakarya) Büyükcoz } \\
\text { Lake }\end{array}$ & Soylu (1991) \\
\hline Alburnus alburnus & Enne Dam Lake & Koyun (2001) \\
\hline Carassius carassius & Enne Dam Lake & Koyun (2001) \\
\hline Carassius auratus & Enne Dam Lake & Koyun (2001) \\
\hline Squalius cephalus & Enne Dam Lake & Koyun (2001) \\
\hline Nemacheilus sp. & Enne Dam Lake & Koyun (2001) \\
\hline Tinca tinca & Kapulukaya Dam Lake & Yildiz (2003) \\
\hline Alburnus nasreddini & Akşehir Lake & Buhurcu (2006) \\
\hline Squalius cephalus & Örenler Dam Lake & Kurupinar (2009) \\
\hline \multicolumn{3}{|l|}{$\begin{array}{l}\text { Pomphorhynchus tereticollis } \\
\text { (Rudolphi, 1809) }\end{array}$} \\
\hline Cobitis bilseli & Beyşehir Lake & Smales et al. (2012) \\
\hline \multicolumn{3}{|l|}{$\begin{array}{l}\text { Pseudoechinorhynchus } \\
\text { clavula Dujardin, } 1845\end{array}$} \\
\hline Cyprinus carpio & $\begin{array}{l}\text { Karasu Brook, Bendimahi } \\
\text { Brook, Engil Brook, Zernek } \\
\text { Dam Lake }\end{array}$ & Topçu (1993) \\
\hline \multicolumn{3}{|l|}{$\begin{array}{l}\text { Triaspiron aphanii Smales, } \\
\text { Aydoğdu, Emre, } 2012\end{array}$} \\
\hline Aphanius mento & Kirkgöz Springs & Smales et al. (2012) \\
\hline
\end{tabular}


Table 3 (continuing): Helminth - Host List.

\begin{tabular}{|c|c|c|}
\hline Phylum Annelida & & \\
\hline $\begin{array}{l}\text { Cystobranchus respirans } \\
\text { (Troschel, 1850) }\end{array}$ & & \\
\hline Salmo trutta fario & Kaz Daği Brook & Geldiay and Balik (1974) \\
\hline $\begin{array}{l}\text { Hemiclepsis marginata } \\
\text { Müller, } 1774\end{array}$ & & \\
\hline Cyprinus carpio & Marmara Lake, Bafa Lake & Geldiay and Balik (1974) \\
\hline Cyprinus carpio & Çapali Lake & Ceylan (2002) \\
\hline Esox lucius & Çapali Lake & Ceylan (2002) \\
\hline $\begin{array}{l}\text { Hirudo medicinalis } \\
\text { Linnaeus, } 1758\end{array}$ & & \\
\hline Esox lucius & Çapali Lake & Ceylan (2002) \\
\hline Cyprinus carpio & Çapali Lake & Ceylan (2002) \\
\hline $\begin{array}{l}\text { Piscicola geometra } \\
\text { Linnaeus, } 1761\end{array}$ & & \\
\hline Rutilus rutilus & Sapanca Lake & Soylu (1990) \\
\hline $\begin{array}{l}\text { Scardinius } \\
\text { erythrophthalmus }\end{array}$ & Sapanca Lake & Soylu (1990) \\
\hline Blicca bjoerkna & Sapanca Lake & Soylu (1990) \\
\hline Tinca tinca & Sapanca Lake & Soylu (1990) \\
\hline Esox lucius & Sapanca Lake & Soylu (1990) \\
\hline Luciobarbus mystaceus & Keban Dam Lake & Sağlam (1992) \\
\hline Tinca tinca & Uluabat Lake & Öztürk (2002) \\
\hline Abramis brama & Terkos Lake & Karatoy (2004) \\
\hline $\begin{array}{l}\text { Scardinius } \\
\text { erythrophthalmus }\end{array}$ & Terkos Lake & Kahveci (2004) \\
\hline Cyprinus carpio & Çavuşçu Lake & Öktener et al. (2007) \\
\hline Carassius gibelio & Uluabat Lake & Emiroğlu and Arslan (2009) \\
\hline Rutilus rutilus & Uluabat Lake & Ceylan et al. (2011) \\
\hline $\begin{array}{l}\text { Trachelobdella torquata } \\
\text { (Grube, 1871) }\end{array}$ & & \\
\hline Carassius carassius & Kovada Lake & Özan and Kir (2005) \\
\hline
\end{tabular}


Table 4: Host - Helminth List.

Silurus glanis Linnaeus, 1758

Ancylodiscoides siluri Zandt, 1924

Ancylodiscoides vistulensis Sivak, 1932

Dactylogyrus vastator (Nybelin, 1924)

Bucephalus polymorphus Baer, 1827

Diplostomum spathaecum (Rudolphi, 1819)

Orientocreadium siluri Bykhovski and Dubi.,1954

Tylodelphys clavata Nordmann, 1832

Bothriocephalus acheilognathi Yamaguti, 1955

Ligula intestinalis Linnaeus, 1758

Proteocephalus osculatus (Goeze, 1782)

Silurotaenia siluri (Batsch, 1786)

Eustrongylides excisus Jagerskiold, 1909

Pomphorhynchus laevis (Müller, 1776)

Sander lucioperca (Linnaeus, 1758)

Ancyrocephalus paradoxus Creplin, 1839

Bucephalus polymorphus Baer, 1827

Clinostomum complanatum Rudolphi, 1819

Tylodelphys clavata Nordmann, 1832

Bothriocephalus acheilognathi Yamaguti, 1955

Caryophyllaeus laticeps (Müller, 1781)

Proteocephalus percae (Müler, 1780)

Eustrongylides excisus Jagerskiold, 1909

\section{Capoeta umbla (Heckel, 1843)}

Dactylogyrus affinis Bychowsky, 1933

Dactylogyrus auriculatus (Nordmann, 1832)

Dactylogyrus extensus Mueller and Cleave, 1932

Dactylogyrus malleus Linstow, 1877

Dactylogyrus minutus Kulwiec, 1927

Bothriocephalus acheilognathi Yamaguti, 1955

Caryophyllaeus laticeps (Müller, 1781)

Khawia armeniaca Cholodkovskii, 1878

Ligula intestinalis Linnaeus, 1758

Philometra intestinalis Dogiel and Bykhovs., 1934

Philometra ovata (Zeder, 1803)

Philometra rischta Skrjabin, 1923

Neoechinorhynchus rutili (Müller, 1780)

Alburnus alburnus (Linnaeus, 1758)

Dactylogyrus alatus Linstow, 1878

Dactylogyrus fraternus Wagener, 1909 
Table 4 (continuing): Host - Helminth List.

Diplozoon homoion Bykhovskii and Nagibi, 1959

Allocreadium isoporum Looss, 1894

Bothriocephalus acheilognathi Yamaguti, 1955

Ligula intestinalis Linnaeus, 1758

Rhabdochona denudata (Dujardin, 1845)

Pomphorhynchus laevis (Müller, 1776)

\section{Chondrostoma regium (Heckel, 1843)}

Dactylogyrus alatus Linstow, 1878

Dactylogyrus elegantis Gusev, 1966

Dactylogyrus extensus Mueller and Cleave, 1932

Dactylogyrus sphyrna Linstow, 1828

Dactylogyrus vistulae Prost, 1957

Gyrodactylus elegans Nordmann, 1832

Gyrodactylus macrocornis Ergens, 1963

Bothriocephalus acheilognathi Yamaguti, 1955

Ligula intestinalis Linnaeus, 1758

Neoechinorhynchus rutili (Müller, 1780)

\section{Alburnus heckeli Battalgil, 1943}

Dactylogyrus alatus Linstow, 1878

\section{Cyprinus carpio Linnaeus, 1758}

Dactylogyrus anchoratus (Dujardin, 1845)

Dactylogyrus ancylostylus Linstow, 1878

Dactylogyrus extensus Mueller and Cleave, 1932

Dactylogyrus minutus Kulwiec, 1927

Dactylogyrus phoxini (Malevitskaya, 1949)

Dactylogyrus vastator (Nybelin, 1924)

Diplozoon homoion Bykhovskii and Nagibi, 1959

Gyrodactylus elegans Nordmann, 1832

Gyrodactylus scardinii Malmberg, 1956

Clinostomum complanatum Rudolphi, 1819

Posthodiplostomum cuticola Nordmann, 1832

Tylodelphys clavata Nordmann, 1832

Bothriocephalus acheilognathi Yamaguti, 1955

Caryophyllaeus brachycollis Janiszewska, 1951

Caryophyllaeus fimbriceps Annenkova-Khlopina, 1919

Caryophyllaeus laticeps (Müller, 1781)

Khawia armeniaca Cholodkovskii, 1878

Ligula intestinalis Linnaeus, 1758

Paradilepis scolecina Rudolphi, 1935

Pseudocapillaria tomentosa (Dujardin, 1843)

Rhabdochona denudata (Dujardin, 1845) 
Table 4 (continuing): Host - Helminth List.

Neoechinorhynchus rutili (Müller, 1780)

Pomphorhynchus laevis (Müller, 1776)

Pseudoechinorhynchus clavula Dujardin, 1845

Hemiclepsis marginata Müller, 1774

Hirudo medicinalis Linnaeus, 1758

Piscicola geometra Linnaeus, 1761

Carassius carassius (Linnaeus, 1758)

Dactylogyrus anchoratus (Dujardin, 1845)

Dactylogyrus minutus Kulwiec, 1927

Dactylogyrus vastator (Nybelin, 1924)

Gyrodactylus katharineri Malmberg, 1964

Pomphorhynchus laevis (Müller, 1776)

\section{Carassius auratus (Linnaeus, 1758)*}

Dactylogyrus anchoratus (Dujardin, 1845)

Gyrodactylus katharineri Malmberg, 1964

Pomphorhynchus laevis (Müller, 1776)

\section{Carassius gibelio (Bloch, 1782)*}

Dactylogyrus anchoratus (Dujardin, 1845)

Dactylogyrus baueri Gussev, 1955

Dactylogyrus extensus Mueller and Cleave, 1932

Dactylogyrus inexpectatus Izjumova, 1955

Dactylogyrus vastator (Nybelin, 1924)

Gyrodactylus scardinii Malmberg, 1956

Piscicola geometra Linnaeus, 1761

\section{Luciobarbus pectoralis (Heckel, 1843)}

Dactylogyrus ancylostylus Linstow, 1878

Dactylogyrus minutus Kulwiec, 1927

Diplozoon barbi Nordman, 1832

Gyrodactylus elegans Nordmann, 1832

Phyllodistomum elongatum Nybelin, 1926

Caryophyllaeus laticeps (Müller, 1781)

Khawia armeniaca Cholodkovskii, 1878

Ligula intestinalis Linnaeus, 1758

Rhabdochona denudata (Dujardin, 1845)

\section{Luciobarbus esocinus Heckel, 1843}

Dactylogyrus ancylostylus Linstow, 1878

Dactylogyrus asper Linstow, 1878

Neoechinorhynchus rutili (Müller, 1780) 
Table 4 (continuing): Host - Helminth List.

Luciobarbus mystaceus (Pallas, 1814)

Dactylogyrus asper Linstow, 1878

Dactylogyrus extensus Mueller and Cleave, 1932

Piscicola geometra Linnaeus, 1761

Rhodeus amarus (Bloch, 1782)

Dactylogyrus bicornis Malevitskaya, 1941

Diplozoon paradoxum Nordman, 1832

Ligula intestinalis Linnaeus, 1758

Luciobarbus escherichii (Steindachner, 1897)

Dactylogyrus carpathicus Zakhvatkin, 1951

Allocreadium isoporum Looss, 1894

Biacetabulum appendiculatum Szidat, 1937

Bothriocephalus acheilognathi Yamaguti, 1955

Caryophyllaeus laticeps (Müller, 1781)

Ligula intestinalis Linnaeus, 1758

Pomphorhynchus laevis (Müller, 1776)

\section{Alburnus chalcoides (Güldenstädt, 1772)}

Dactylogyrus chalcalburni Dogiel and Bychowsky 1833

Diplozoon paradoxum Nordman, 1832

Paradiplozoon megan Bykhovskii and Nagibi, 1959

Posthodiplostomum cuticola Nordmann, 1832

Bothriocephalus acheilognathi Yamaguti, 1955

\section{Blicca bjoerkna (Linnaeus, 1758)}

Dactylogyrus cornu Linstow, 1878

Dactylogyrus cornoides (Gläser and Gussev, 1971)

Dactylogyrus crucifer Wagener, 1857

Dactylogyrus difformoides Glaser and Gusev, 1967

Dactylogyrus distinguendus (Linstow, 1878)

Dactylogyrus falcatus (Wedl, 1857)

Dactylogyrus sphyrna Linstow, 1828

Dactylogyrus wunderi Bykhovskii, 1931

Aspidogaster limacoides Diesing, 1835

Asymphylodora imitans (Mühling, 1898)

Diplostomum spathaecum (Rudolphi, 1819)

Posthodiplostomum cuticola Nordmann, 1832

Tylodelphys clavata Nordmann, 1832

Bothriocephalus acheilognathi Yamaguti, 1955

Caryophyllaeus laticeps (Müller, 1781)

Piscicola geometra Linnaeus, 1761 
Table 4 (continuing): Host - Helminth List.

\begin{tabular}{|l|}
\hline Vimba vimba (Linnaeus, 1758) \\
\hline Dactylogyrus cornu Linstow, 1878 \\
\hline Dactylogyrus cornoides (Gläser and Gussev, 1971) \\
\hline Dactylogyrus sphyrna Linstow, 1828 \\
\hline Aspidogaster limacoides Diesing, 1835 \\
\hline Posthodiplostomum cuticola Nordmann, 1832 \\
\hline Tylodelphys clavata Nordmann, 1832 \\
\hline Caryophyllaeus laticeps (Müller, 1781) \\
\hline Ligula intestinalis Linnaeus, 1758 \\
\hline Neoechinorhynchus rutili (Müller, 1780) \\
\hline
\end{tabular}

\section{Rutilus frisii (Nordmann, 1840)}

Dactylogyrus crucifer Wagener, 1857

Dactylogyrus frisii Bychowsky, 1933

Dactylogyrus nybelini Markewitsch, 1933

Bothriocephalus acheilognathi Yamaguti, 1955

Caryophyllaeus laticeps (Müller, 1781)

Neoechinorhynchus rutili (Müller, 1780)

\section{Rutilus rutilus (Linnaeus, 1758)}

Dactylogyrus crucifer Wagener, 1857

Dactylogyrus sphyrna Linstow, 1828

Dactylogyrus vistulae Prost, 1957

Diplozoon homoion Bykhovskii and Nagibi, 1959

Diplozoon paradoxum Nordman, 1832

Aspidogaster limacoides Diesing, 1835

Diplostomum spathaecum (Rudolphi, 1819)

Opisthorchis felinus (Rivolta, 1884)

Tylodelphys clavata Nordmann, 1832

Bothriocephalus acheilognathi Yamaguti, 1955

Caryophyllaeides fennica (Schneider, 1902)

Ligula intestinalis Linnaeus, 1758

Piscicola geometra Linnaeus, 1761

\section{Capoeta tinca (Heckel, 1843)}

Dactylogyrus crucifer Wagener, 1857

Gyrodactylus narzikulovi Ergens and Dzhalilov, 1979

Clinostomum complanatum Rudolphi, 1819

Ligula intestinalis Linnaeus, 1758

\section{Scardinius erythrophthalmus (Linnaeus, 1758)}

Dactylogyrus difformis Wagener, 1857

Dactylogyrus difformoides Glaser and Gusev, 1967 
Table 4 (continuing): Host - Helminth List.

\begin{tabular}{|l}
\hline Dactylogyrus izjumovae Gusev, 1966 \\
\hline Dactylogyrus sphyrna Linstow, 1828 \\
\hline Asymphylodora markewitschi Kulakovskaya, 1947 \\
\hline Clinostomum complanatum Rudolphi, 1819 \\
\hline Diplostomum spathaecum (Rudolphi, 1819) \\
\hline Posthodiplostomum cuticola Nordmann, 1832 \\
\hline Tylodelphys clavata Nordmann, 1832 \\
\hline Tetracotyle percae-fluviatilis (Linstow, 1856) \\
\hline Caryophyllaeides fennica (Schneider, 1902) \\
\hline Ligula intestinalis Linnaeus, 1758 \\
\hline Eustrongylides excisus Jagerskiold, 1909 \\
\hline Skrjabillanus scardinii Molnár, 1966 \\
\hline Piscicola geometra Linnaeus, 1761 \\
\hline
\end{tabular}

\footnotetext{
Abramis brama (Linnaeus, 1758)

Dactylogyrus distinguendus (Linstow, 1878)

Dactylogyrus sphyrna Linstow, 1828

Diplostomum spathaecum (Rudolphi, 1819)

Tylodelphys clavata Nordmann, 1832

Tetracotyle percae-fluviatilis (Linstow, 1856)
}

\section{Squalius cephalus (Linnaeus, 1758)}

Dactylogyrus elegantis Gusev, 1966

Dactylogyrus extensus Mueller and Cleave, 1932

Dactylogyrus folkmanovae Ergens, 1956

Dactylogyrus naviculoides Ergens, 1960

Dactylogyrus prostae Molnar, 1964

Dactylogyrus vistulae Prost, 1957

Paradiplozoon megan Bykhovskii and Nagibi, 1959

Allocreadium isoporum Looss, 1894

Bothriocephalus acheilognathi Yamaguti, 1955

Khawia armeniaca Cholodkovskii, 1878

Ligula intestinalis Linnaeus, 1758

Philometra ovata (Zeder, 1803)

Rhabdochona denudata (Dujardin, 1845)

Pomphorhynchus laevis (Müller, 1776)

\section{Tinca tinca (Linnaeus, 1758)}

Dactylogyrus macrocanthus Wagener, 1857

Gyrodactylus medius Kathariner, 1894

Asymphylodora tincae (Mooder, 1790)

Diplostomum spathaecum (Rudolphi, 1819)

Tylodelphys clavata Nordmann, 1832

Bothriocephalus acheilognathi Yamaguti, 1955 
Table 4 (continuing): Host - Helminth List.

Caryophyllaeus laticeps (Müller, 1781)

Ligula intestinalis Linnaeus, 1758

Proteocephalus torulosus (Batsch, 1786)

Acanthocephalus anguillae Müller, 1780

Pomphorhynchus laevis (Müller, 1776)

Piscicola geometra Linnaeus, 1761

\section{Barbus plebejus Bonaparte, 1839}

Dactylogyrus malleus Linstow, 1877

Gyrodactylus elegans Nordmann, 1832

Gyrodactylus hemibarbi (Ergens, 1980)

Allocreadium isoporum Looss, 1894

Ligula intestinalis Linnaeus, 1758

Rhabdochona denudata (Dujardin, 1845)

Pseudophoxinus antalyae Bogutskaya, 1992

Dactylogyrus ergensi Molnar, 1964

Dactylogyrus sphyrna Linstow, 1828

Diplozoon homoion Bykhovskii and Nagibi, 1959

Eustrongylides excisus Jagerskiold, 1909

\section{Barbus grypus Heckel, 1843}

Dactylogyrus extensus Mueller and Cleave, 1932

\section{Capoeta capoeta (Güldenstädt, 1773)}

Dactylogyrus pulcher Bychowsky, 1957

Dactylogyrus vastator (Nybelin, 1924)

Caryophyllaeus laticeps (Müller, 1781)

Ligula intestinalis Linnaeus, 1758

Rhabdochona denudata (Dujardin, 1845)

\section{Esox lucius Linnaeus, 1758}

Tetraonchus monenteron Diesing, 1858

Asymphylodora tincae (Mooder, 1790)

Bucephalus polymorphus Baer, 1827

Diplostomum spathaecum (Rudolphi, 1819)

Diplodiscus subclavatus Diesing, 1836

Rhipidocotyle fennica Taskinen, 1991

Tylodelphys clavata Nordmann, 1832

Bathybothrium rectangulum (Bloch, 1782)

Ligula intestinalis Linnaeus, 1758

Triaenophorus crassus Forel, 1880

Camallanus truncatus (Rudolphi, 1814) 
Table 4 (continuing): Host - Helminth List.

Raphidascaris acus (Bloch, 1779)

Acanthocephalus anguillae Müller, 1780

Neoechinorhynchus rutili (Müller, 1780)

Pomphorhynchus laevis (Müller, 1776)

Hemiclepsis marginata Müller, 1774

Hirudo medicinalis Linnaeus, 1758

Piscicola geometra Linnaeus, 1761

\section{Garra rufa (Heckel, 1843)}

Dactylogyrus rectotrabus Gussev, Jalali and Molnar, 1993

Ligula intestinalis Linnaeus, 1758

Capoeta trutta (Heckel, 1843)

Diplozoon barbi Nordman, 1832

Bothriocephalus acheilognathi Yamaguti, 1955

Khawia armeniaca Cholodkovskii, 1878

Neoechinorhynchus rutili (Müller, 1780)

Rutilus rubilio (Bonaparte, 1837)

Dactylogyrus sphyrna Linstow, 1828

Neoechinorhynchus rutili (Müller, 1780)

\section{Aphanius sp.}

Dactylogyrus vastator (Nybelin, 1924)

Alburnus orontis Sauvage, 1882

Dactylogyrus vastator (Nybelin, 1924)

Ligula intestinalis Linnaeus, 1758

\section{Acantobrama marmid Heckel, 1843}

Diplozoon paradoxum Nordman, 1832

Bothriocephalus acheilognathi Yamaguti, 1955

Caryophyllaeus laticeps (Müller, 1781)

Khawia armeniaca Cholodkovskii, 1878

Ligula intestinalis Linnaeus, 1758

\section{Capoeta damascina (Valenciennes, 1842)}

Dogielius forceps Bychowsky, 1936

Monobothrium auriculatum Kulakovskaya, 1961

\section{Gasterosteus aculeatus Linnaeus, 1758*}

Gyrodactylus arcuatus Bychowsky, 1933 
Table 4 (continuing): Host - Helminth List.

Cobitis simplicispina Hankó, 1925

Gyrodactylus cobitis Bychowsky, 1933

Neogobius fluviatilis (Pallas, 1814)*

Gyrodactylus gobii Shul'man, 1953

Bothriocephalus acheilognathi Yamaguti, 1955

Ligula pavlovskii Dubinina, 1959

Eustrongylides excisus Jagerskiold, 1909

Neogobius melanostomus (Pallas, 1814)*

Gyrodactylus proterorhini Ergens, 1967

Pygidiopsis genata Looss, 1907

Neoechinorhynchus rutili (Müller, 1780)

Lepomis gibbosus (Linnaeus, 1758)*

Onchocleidus similis Mueller, 1936

Clinostomum complanatum Rudolphi, 1819

Tylodelphys clavata Nordmann, 1832

Clarias gariepinus (Burchell, 1822)

Quadriacanthus clariadis (Paperna, 1961)

Orientocreadium batrachoides Tubangui, 1931

Polyonchobothrium magnum (Zme’ev, 1936)

\section{Alburnus sp.}

Asymphylodora tincae (Mooder, 1790)

Clinostomum complanatum Rudolphi, 1819

Ligula intestinalis Linnaeus, 1758

Neoechinorhynchus rutili (Müller, 1780)

Pomphorhynchus laevis (Müller, 1776)

Barbus sp.

Asymphylodora tincae (Mooder, 1790)

Neoechinorhynchus rutili (Müller, 1780)

Knipowitschia caucasica (Berg, 1916)

Bucephalus polymorphus Baer, 1827

\section{Chondrostoma sp.}

Clinostomum complanatum Rudolphi, 1819

Philometra intestinalis Dogiel and Bykhowsky, 1934 
Table 4 (continuing): Host - Helminth List.

Varcorhinus sp.

Clinostomum complanatum Rudolphi, 1819

Perca fluviatilis Linnaeus, 1758

Clinostomum complanatum Rudolphi, 1819

Tylodelphys clavata Nordmann, 1832

Proteocephalus percae (Müler, 1780)

Eustrongylides excisus Jagerskiold, 1909

Salmo trutta abanticus Tortonese, 1954

Crepidostomum farionis (Muller, 1780)

\section{Salmo trutta fario Linnaeus, 1758}

Posthodiplostomum minimum Maccallum, 1921

Cystobranchus respirans (Troschel, 1850)

\section{Atherina boyeri Risso, 1810*}

Tylodelphys clavata Nordmann, 1832

Bothriocephalus acheilognathi Yamaguti, 1955

Eustrongylides excisus Jagerskiold, 1909

\section{Alburnus mossulensis Heckel, 1843}

Bothriocephalus acheilognathi Yamaguti, 1955

Ligula intestinalis Linnaeus, 1758

\section{Capoeta bergamae Karaman, 1969}

Ligula intestinalis Linnaeus, 1758

Alburnoides bipunctatus (Bloch, 1782)

Ligula intestinalis Linnaeus, 1758

Chondrostoma nasus (Linnaeus, 1758)

Ligula intestinalis Linnaeus, 1758

Alburnus escherichii Steindachner, 1897

Ligula intestinalis Linnaeus, 1758

Gobio gobio (Linnaeus, 1758)

Ligula intestinalis Linnaeus, 1758

\section{Aphanius mento (Heckel, 1843)}

Eustrongylides excisus Jagerskiold, 1909

Triaspiron aphanii Smales, Aydoğdu and Emre, 2012 
Table 4 (continuing): Host - Helminth List.

Pseudophoxinus battalgilae Bogutskaya, 1997

Eustrongylides excisus Jagerskiold, 1909

\section{Squalius lepidus Heckel, 1843}

Molnaria intestinalis Dogiel and Bychowsky, 1934

Capoeta antalyensis (Battalgil, 1943)

Rhabdochona denudata (Dujardin, 1845)

Luciobarbus mursa (Güldenstädt, 1773)

Rhabdochona denudata (Dujardin, 1845)

\section{Aphanius chantrei (Gaillard, 1895)}

Spiroxys contortus Rudolphi, 1819

Neoechinorhynchus rutili (Müller, 1780)

\section{Aphanius danfordii (Boulenger, 1890)}

Spiroxys contortus Rudolphi, 1819

Neoechinorhynchus rutili (Müller, 1780)

\section{Capoeta barroisi Lortet, 1894}

Neoechinorhynchus zabensis Amin, Abdullah and Mhaisen, 2003

Nemacheilus sp.

Pomphorhynchus laevis (Müller, 1776)

\section{Alburnus nasreddini Battalgil, 1943}

Pomphorhynchus laevis (Müller, 1776)

\section{Cobitis bilseli Battalgil, 1942}

Pomphorhynchus tereticollis (Rudolphi, 1809)

\section{Silurus triostegus Heckel, 1843}

Senga mastacembeli Rahemo, 1996

Atherina boyeri introduced into several western lakes and Beyșehir Lake - Lepomis gibbosus, Carassius gibelio, Carassius auratus are not native to Turkey, they were introduced into the lakes and rivers.

Gasterosteus aculeatus is distributed in the Black Sea, Marmara Sea, Aegean Sea and Mediterranean Sea and adjacent watersheds; Neogobius fluviatilis in the Black Sea watersheds; Neogobius melanostomus in the Black Sea, Marmara Sea, Aegean Sea and the Caspian Sea watersheds. 
Table 5: Unnamed helminth species (at genera level) on freshwater fishes of Turkey.

\begin{tabular}{|c|c|c|}
\hline \multicolumn{3}{|l|}{ Phylum Platyhelminthes } \\
\hline \multicolumn{3}{|l|}{ Class Monogenea } \\
\hline \multicolumn{3}{|l|}{ Ancyrocephalus sp. } \\
\hline Aphanius chantrei & Sirakaraağaçlar Stream & Özer (2006) \\
\hline \multicolumn{3}{|l|}{ Paradiplozoon sp. } \\
\hline Abramis brama & Terkos Lake & Soylu (2009) \\
\hline Blicca bjoerkna & Sapanca Lake & Soylu (2012) \\
\hline Cyprinus carpio & Siğirci Lake & Çolak (2013) \\
\hline \multicolumn{3}{|l|}{ Cleidodiscus sp. } \\
\hline Silurus glanis & Gölbasi Lake & Ekingen (1976) \\
\hline \multicolumn{3}{|l|}{ Diplozoon sp. } \\
\hline Scardinius erythrophthalmus & Karacabey Lagoon Lake & Öztürk et al. (2002) \\
\hline \multicolumn{3}{|l|}{ Dactylogyrus sp. } \\
\hline Tinca tinca & Gölbaşi Lake, Eymir Lake & Burgu et al. (1988) \\
\hline Cyprinus carpio & $\begin{array}{l}\text { Gölbaşi Lake, Eymir Lake, } \\
\text { Çifteler-Sakaryabaşi Fish } \\
\text { Production Station, } \\
\text { Kurtboğazi Dam Lake, } \\
\text { Sariyar Dam Lake, } \\
\text { Kizilcahamam Brook, } \\
\text { Hirfanli Dam Lake, Çankiri- } \\
\text { Günerdiğin Pond }\end{array}$ & Burgu et al. (1988) \\
\hline Alburnus sp. & $\begin{array}{l}\text { Gölbaşi Lake, Eymir Lake, } \\
\text { Çifteler-Sakaryabaşi Fish } \\
\text { Production Station, } \\
\text { Kurtboğazi Dam Lake, } \\
\text { Sariyar Dam Lake, } \\
\text { Kizilcahamam Brook }\end{array}$ & Burgu et al. (1988) \\
\hline Silurus glanis & $\begin{array}{l}\text { Gölbaşi Lake, Eymir Lake, } \\
\text { Sariyar Dam Lake }\end{array}$ & Burgu et al. (1988) \\
\hline Barbus sp. & $\begin{array}{l}\text { Çifteler-Sakaryabaşi Fish } \\
\text { Production Station, Sariyar } \\
\text { Dam Lake }\end{array}$ & Burgu et al. (1988) \\
\hline Chondrostoma sp. & $\begin{array}{l}\text { Kizilcahamam Brook, } \\
\text { Nallihan Brook }\end{array}$ & Burgu et al. (1988) \\
\hline Varicorhinus sp. & $\begin{array}{l}\text { Çifteler-Sakaryabaşi Fish } \\
\text { Production Station, } \\
\text { Kurtboğazi Dam Lake, } \\
\text { Çankiri-Günerdiğin Pond, } \\
\text { Nallihan Brook, } \\
\text { Kizilcahamam Brook }\end{array}$ & Burgu et al. (1988) \\
\hline
\end{tabular}


Table 5 (continuing): Unnamed helminth species on freshwater fishes of Turkey.

\begin{tabular}{|c|c|c|}
\hline Cyprinus carpio & $\begin{array}{l}\text { Kocadere Lake, Uluabat } \\
\text { Lake, Ekinli Lagoon Lake }\end{array}$ & Altin (1989) \\
\hline Cyprinus carpio & $\begin{array}{l}\text { Kocadere Lake, Uluabat } \\
\text { Lake, Ekinli Lagoon Lake }\end{array}$ & Oğuz (1991) \\
\hline Tinca tinca & İznik Lake & Aydoğdu et al. (1996a) \\
\hline Tinca tinca & Mogan Lake & Sönmez (1996) \\
\hline Cyprinus carpio & Mogan Lake & Sönmez (1996) \\
\hline Alburnus escherichii & Mogan Lake & Sönmez (1996) \\
\hline Cyprinus carpio & $\begin{array}{l}\text { Çifteler-Sakaryabaşi Fish } \\
\text { Production Sitation }\end{array}$ & Atay et al. (1999) \\
\hline Vimba vimba & Sirakaraağaçlar Stream & Ünsal (2008) \\
\hline Cyprinus carpio & Halil-ür Rahman Lake & Pişkin and Ütük (2008) \\
\hline Capoeta capoeta & Almus Dam Lake & Özgül (2008) \\
\hline \multicolumn{3}{|l|}{ Gyrodactylus sp. } \\
\hline Salmo trutta fario & İstanbul Brook & Soylu (1985) \\
\hline Cyprinus carpio & $\begin{array}{l}\text { Gölbaşi Lake, Eymir Lake, } \\
\text { Çifteler-Sakaryabaşi Fish } \\
\text { Production Station, } \\
\text { Kurtboğazi Dam Lake, } \\
\text { Sariyar Dam Lake, } \\
\text { Kizilcahamam Brook, } \\
\text { Hirfanli Dam Lake, Çankiri- } \\
\text { Günerdiğin Pond }\end{array}$ & Burgu et al. (1988) \\
\hline Alburnus sp. & $\begin{array}{l}\text { Gölbaşi Lake, Eymir Lake, } \\
\text { Çifteler-Sakaryabaşi Fish } \\
\text { Production Station, } \\
\text { Kurtboğazi Dam Lake, } \\
\text { Sariyar Dam Lake, } \\
\text { Kizilcahamam Brook }\end{array}$ & Burgu et al. (1988) \\
\hline Chondrostoma sp. & $\begin{array}{l}\text { Kizilcahamam Brook, } \\
\text { Nallihan Brook }\end{array}$ & Burgu et al. (1988) \\
\hline Varicorhinus sp. & $\begin{array}{l}\text { Çifteler-Sakaryabaşi Fish } \\
\text { Production Station, } \\
\text { Kurtboğazi Dam Lake, } \\
\text { Çankiri-Günerdiğin Pond, } \\
\text { Kizilcahamam Brook, } \\
\text { Nallihan Brook }\end{array}$ & Burgu et al. (1988) \\
\hline Barbus sp. & $\begin{array}{l}\text { Gölbaşi Lake, Eymir Lake, } \\
\text { Sariyar Dam Lake }\end{array}$ & Burgu et al. (1988) \\
\hline Cyprinus carpio & Bekteşağa Pond & Özer (1995) \\
\hline Aphanius chantrei & Sarikum Lagoon Lake & Öztürk (2005) \\
\hline Cyprinus carpio & Sapanca Lake & Uzunay (2006) \\
\hline Chondrostoma regium & Almus Dam Lake & Özgül and Turgut (2006) \\
\hline
\end{tabular}


Table 5 (continuing): Unnamed helminth species on freshwater fishes of Turkey.

\begin{tabular}{|c|c|c|}
\hline Aphanius chantrei & Sirakaraağaçlar Stream & Özer (2006) \\
\hline Aphanius danfordii & Sarikum Lagoon Lake & Öztürk and Özer (2008a) \\
\hline Aphanius danfordii & Sirakaraağaçlar Stream & Ünsal (2008) \\
\hline Neogobius melanostomus & Sirakaraağaçlar Stream & Ünsal (2008) \\
\hline Capoeta capoeta & Almus Dam Lake & Özgül (2008) \\
\hline Capoeta tinca & Almus Dam Lake & Özgül (2008) \\
\hline Cyrinus carpio & Emre Dam Lake & Öztürk (2011) \\
\hline Carassius auratus & Emre Dam Lake & Öztürk (2011) \\
\hline Carassius carassius & Emre Dam Lake & Öztürk (2011) \\
\hline Blicca bjoerkna & Sapanca Lake & Soylu (2012) \\
\hline Perca fluviatilis & Siğirci Lake & Soylu (2013) \\
\hline Cyprinus carpio & Siğirci Lake & Çolak (2013) \\
\hline Carassius gibelio & Siğirci Lake & Çolak (2013) \\
\hline Sander lucioperca & Siğirci Lake & Çolak (2013) \\
\hline Lepomis gibbosus & Siğirci Lake & Çolak (2013) \\
\hline \multicolumn{3}{|l|}{ Salsuginus sp. } \\
\hline Aphanius chantrei & Sarikum Lagoon Lake & Öztürk (2005) \\
\hline Aphanius danfordii & Sarikum Lagoon Lake & Öztürk and Özer (2008a) \\
\hline Aphanius danfordii & Sirakaraağaçlar Stream & Ünsal (2008) \\
\hline \multicolumn{3}{|l|}{ Tetraonchus sp. } \\
\hline Esox lucius & Mogan Lake & Sönmez (1996) \\
\hline Acantobrama marmid & Tigem Reservoirs & Zeren (2008) \\
\hline \multicolumn{3}{|l|}{ Phylum Platyhelminthes } \\
\hline \multicolumn{3}{|l|}{ Class Digenea } \\
\hline \multicolumn{3}{|l|}{ Ascocotyle sp. } \\
\hline Aphanius chantrei & Sarikum Lagoon Lake & Öztürk (2005) \\
\hline Aphanius chantrei & Sirakaraağaçlar Stream & Özer (2006) \\
\hline Neogobius melanostomus & Sirakaraağaçlar Stream & Özer (2007) \\
\hline Aphanius danfordii & Sarikum Lagoon Lake & Öztürk and Özer (2008a) \\
\hline \multicolumn{3}{|l|}{ Digenea sp. } \\
\hline Tinca tinca & $\begin{array}{l}\text { Mogan Lake, Hirfanli Dam } \\
\text { Lake, Kizilcahamam Brook }\end{array}$ & Erkul (1997) \\
\hline Tinca tinca & Kapulukaya Dam Lake & Yildiz (2003) \\
\hline Neogobius melanostomus & $\begin{array}{l}\text { Sirakaraağaçlar } \\
\text { Stream }\end{array}$ & Özer (2007) \\
\hline \multicolumn{3}{|l|}{ Diplostomum sp. } \\
\hline Blicca bjoerkna & Uluabat Lake & Akinci (1999) \\
\hline Acanthobrama marmid & Keban Dam Lake & Dörücü and İspir (2001) \\
\hline Silurus glanis & Terkos Lake & Soylu (2005) \\
\hline
\end{tabular}


Table 5 (continuing): Unnamed helminth species on freshwater fishes of Turkey.

\begin{tabular}{|c|c|c|}
\hline Carassius carassius & $\begin{array}{l}\text { Kepez I Hydro Electric } \\
\text { Power Plant Loading Pond }\end{array}$ & Soylu and Emre (2005) \\
\hline Luciobarbus esocinus & Keban Dam Lake & Dörücü and İspir (2005) \\
\hline Capoeta umbla & Keban Dam Lake & Dörücü and İspir (2005) \\
\hline Chondrostoma regium & Keban Dam Lake & Dörücü and İspir (2005) \\
\hline Squalius cephalus & Keban Dam Lake & Dörücü and İspir (2005) \\
\hline Blicca bjoerkna & Sapanca Lake & Soylu (2006) \\
\hline Rutilus rutilus & Sapanca Lake & Karabiber (2006) \\
\hline Tinca tinca & Sapanca Lake & Akbeniz (2006) \\
\hline Cyprinus carpio & Sapanca Lake & Uzunay (2006) \\
\hline Vimba vimba & Sapanca Lake & Uzunay (2006) \\
\hline Cyprinus carpio & Sapanca Lake & Uzunay (2006) \\
\hline Chondrostoma regium & Almus Dam Lake & Özgül and Turgut (2006) \\
\hline Alburnus alburnus & Mustafakemalpaşa Stream & Aydoğdu and Selver (2006) \\
\hline Vimba vimba & Gölbaşi Dam Lake & Aydoğdu et al. (2008) \\
\hline Capoeta trutta & Keban Dam Lake & Dörücü et al. (2008) \\
\hline Cyprinus carpio & Almus Dam Lake & Özgül (2008) \\
\hline Capoeta capoeta & Almus Dam Lake & Özgül (2008) \\
\hline Capoeta tinca & Almus Dam Lake & Özgül (2008) \\
\hline Squalius cephalus & Örenler Dam Lake & Kurupinar (2009) \\
\hline Cyprinus carpio & Keban Dam Lake & Karabulut (2009) \\
\hline Perca fluviatilis & Siğirci Lake & Soylu (2013) \\
\hline Atherina boyeri & İznik Lake & Çolak (2013) \\
\hline Cyprinus carpio & Siğirci Lake & Çolak (2013) \\
\hline Carassius gibelio & Siğgirci Lake & Çolak (2013) \\
\hline Sander lucioperca & Siğirci Lake & Çolak (2013) \\
\hline Lepomis gibbosus & Siğirci Lake & Çolak (2013) \\
\hline Scardinius erythropthalmus & Siğirci Lake & Çolak (2013) \\
\hline Rutilus rutilus & Siğirci Lake & Çolak (2013) \\
\hline Esox lucius & Siğirci Lake & Çolak (2013) \\
\hline Silurus glanis & Siğirci Lake & Çolak (2013) \\
\hline \multicolumn{3}{|l|}{ Diplodiscus sp. } \\
\hline Esox lucius & Uluabat Lake & Öztürk (1995) \\
\hline \multicolumn{3}{|l|}{ Neascus sp. } \\
\hline Salmo trutta fario & Munzur Stream & Ekingen (1975) \\
\hline \multicolumn{3}{|l|}{ Orientocreadium sp. } \\
\hline Clarias gariepinus & $\begin{array}{l}\text { Kepez I Hydro Electric } \\
\text { Power Plant Loading Pond }\end{array}$ & Soylu and Emre (2005) \\
\hline \multicolumn{3}{|l|}{ Posthodiplostomum sp. } \\
\hline Aphanius chantrei & Sarikum Lagoon Lake & Öztürk (2005) \\
\hline
\end{tabular}


Table 5 (continuing): Unnamed helminth species on freshwater fishes of Turkey.

\begin{tabular}{|c|c|c|}
\hline Aphanius chantrei & Sirakaraağaçlar Stream & Özer (2006) \\
\hline Aphanius danfordii & Sarikum Lagoon Lake & Öztürk and Özer (2008a) \\
\hline \multicolumn{3}{|l|}{ Rhipidocotyle sp. } \\
\hline Esox lucius & Uluabat Lake & Öztürk (1995) \\
\hline \multicolumn{3}{|l|}{ Sanguinicola sp. } \\
\hline Cyprinus carpio & $\begin{array}{l}\text { Gölbaşi Lake, Eymir Lake, } \\
\text { Çifteler-Sakaryabaşi Fish } \\
\text { Production Station, } \\
\text { Kurtboğazi Dam Lake, } \\
\text { Sariyar Dam Lake, } \\
\text { Kizilcahamam Brook, } \\
\text { Hirfanli Dam Lake, Çankiri- } \\
\text { Günerdiğin Pond }\end{array}$ & Burgu et al. (1988) \\
\hline \multicolumn{3}{|l|}{ Tetracotyle sp. } \\
\hline Vimba vimba & Sapanca Lake & Uzunay (2006) \\
\hline Blicca bjoerkna & Sapanca Lake & Soylu (2006) \\
\hline Perca fluviatilis & Siğirci Lake & Soylu (2013) \\
\hline Sander lucioperca & Siğirci Lake & Çolak (2013) \\
\hline Lepomis gibbosus & Siğirci Lake & Çolak (2013) \\
\hline \multicolumn{3}{|l|}{ Creptotrema sp. } \\
\hline Salmo trutta fario & Munzur Stream & Ekingen (1975) \\
\hline \multicolumn{3}{|c|}{ Phylum Platyhelminthes } \\
\hline \multicolumn{3}{|c|}{ Class Cestoda } \\
\hline \multicolumn{3}{|l|}{ Bothriocephalus sp. } \\
\hline Cyprinus carpio & Uluabat Lake & Oğuz et al. (1996a) \\
\hline Esox lucius & Mogan Lake & Sönmez (1996) \\
\hline Cyprinus carpio & Mogan Lake & Sönmez (1996) \\
\hline Alburnus escherichii & Mogan Lake & Sönmez (1996) \\
\hline Cyprinus carpio & İznik Lake & Aydoğdu (1997) \\
\hline \multicolumn{3}{|l|}{ Caryophyllaeus sp. } \\
\hline Cyprinus carpio & Porsuk Stream & Yetim (1985) \\
\hline Cyprinus carpio & Seyhan River & Cengizler et al. (2001) \\
\hline \multicolumn{3}{|l|}{ Caryophyllaeides sp. } \\
\hline Blicca bjoerkna & Uluabat Lake & Akinci (1999) \\
\hline
\end{tabular}


Table 5 (continuing): Unnamed helminth species on freshwater fishes of Turkey.

\begin{tabular}{|c|c|c|}
\hline \multicolumn{3}{|l|}{ Cyclophyllidea sp. } \\
\hline & $\begin{array}{l}\text { Çifteler-Sakaryabaşi Fish } \\
\text { Production Station, } \\
\text { Kurtboğazi Lake, Çankiri- } \\
\text { Günerdiğin Pond, Nallihan } \\
\text { and Kizilcahamam brooks }\end{array}$ & Burgu et al. (1988) \\
\hline \multicolumn{3}{|l|}{ Diphyllobothrium sp. } \\
\hline Capoeta umbla & Hazar Lake & Aksoy (1996) \\
\hline \multicolumn{3}{|l|}{ Ligula sp. } \\
\hline Vimba vimba & Porsuk River & Yetim (1985) \\
\hline Tinca tinca & Mogan Lake & Öğe and Aydin (1995) \\
\hline Esox lucius & Mogan Lake & Sönmez (1996) \\
\hline Cyprinus carpio & Mogan Lake & Sönmez (1996) \\
\hline Alburnus escherichii & Mogan Lake & Sönmez (1996) \\
\hline Silurus glanis & İznik Lake & Aydoğdu et al. (1996b) \\
\hline Tinca tinca & Kapulukaya Dam Lake & Yildiz (2003) \\
\hline \multicolumn{3}{|l|}{ Proteocephalus sp. } \\
\hline Cyprinus carpio & Porsuk Stream & Yetim (1985) \\
\hline Aphanius chantrei & Sirakaraağaçlar Stream & Özer (2006) \\
\hline \multicolumn{3}{|l|}{ Schistocephalus sp. } \\
\hline Cyprinus carpio & Seyhan River & Cengizler et al. (2001) \\
\hline Luciobarbus pectoralis & Seyhan River & Şahan and Cengizler (2003) \\
\hline Tinca tinca & Gölbaşi Lake, Eymir Lake & Burgu et al. (1988) \\
\hline Tinca tinca & $\begin{array}{l}\text { Mogan Lake, Hirfanli Dam } \\
\text { Lake, Kizilirmak Brook }\end{array}$ & Erkul (1997) \\
\hline Cyprinus carpio & Mogan Lake & Erkul (1997) \\
\hline Alburnus escherichii & Mogan Lake & Erkul (1997) \\
\hline Esox lucius & Mogan Lake & Erkul (1997) \\
\hline Tinca tinca & Kapulukaya Dam Lake & Yildiz (2003) \\
\hline Esox lucius & Siğirci Lake & Çolak (2013) \\
\hline Silurus glanis & Siğirci Lake & Çolak (2013) \\
\hline \multicolumn{3}{|l|}{ Philonema sp. } \\
\hline Salmo trutta fario & Munzur Stream & Ekingen (1975) \\
\hline \multicolumn{3}{|l|}{ Raphidascaris sp. } \\
\hline Esox lucius & Çapali Lake & Ceylan (2002) \\
\hline
\end{tabular}


Table 5 (continuing): Unnamed helminth species on freshwater fishes of Turkey.

\begin{tabular}{|c|c|c|}
\hline \multicolumn{3}{|l|}{ Rhabdochona sp. } \\
\hline Cyprinion macrostomum & Balikli Thermal & Saygi and Bardakçi (1990) \\
\hline Gara rufa & Topardic Stream & Saygi and Bardakçi (1990) \\
\hline Scardinius erythrophthalmus & Uluabat Lake & Oğuz and Öztürk (1993) \\
\hline Alburnus chalcoides & Tödürge Lake & Yildirim and Ünver (2006) \\
\hline \multicolumn{3}{|l|}{ Spiroxys sp. } \\
\hline Aphanius chantrei & Sirakaraağaçlar Stream & Özer (2006) \\
\hline Neogobius melanostomus & Sirakaraağaçlar Stream & Özer (2007) \\
\hline \multicolumn{3}{|l|}{ Phylum Acanthocephala } \\
\hline \multicolumn{3}{|l|}{ Acanthocephalus sp. } \\
\hline Salmo trutta fario & Munzur Stream & Ekingen (1975) \\
\hline \multicolumn{3}{|l|}{ Neoechinorhynchus sp. } \\
\hline Salmo trutta fario & Munzur Stream & Ekingen (1976) \\
\hline Luciobarbus pectoralis & Keban Dam Lake & $\begin{array}{l}\text { Özdemir and Sarieyyüpoğlu } \\
\text { (1993) }\end{array}$ \\
\hline Capoeta capoeta & Murat River, Aras River & Aslan (2009) \\
\hline Capoeta barroisi & Murat River, Aras River & Aslan (2009) \\
\hline Barbus plebejus & Murat River, Aras River & Aslan (2009) \\
\hline Cyprinus carpio & Siğirci Lake & Çolak (2013) \\
\hline Esox lucius & Siğirci Lake & Çolak (2013) \\
\hline \multicolumn{3}{|l|}{ Paulisentis sp. } \\
\hline Aphanius chantrei & Sirakaraağaçlar Stream & Özer (2006) \\
\hline \multicolumn{3}{|l|}{ Pomphorhynchus sp. } \\
\hline Chondrostoma nasus & Porsuk Stream & Yetim (1985) \\
\hline Luciobarbus escherichii & Porsuk Stream & Yetim (1985) \\
\hline Capoeta capoeta & Murat River, Aras River & Aslan (2009) \\
\hline Barbus plebejus & Murat River, Aras River & Aslan (2009) \\
\hline Squalius cephalus & Murat River, Aras River & Aslan (2009) \\
\hline \multicolumn{3}{|l|}{ Phylum Annelida } \\
\hline \multicolumn{3}{|l|}{ Actinobdella sp. } \\
\hline Luciobarbus mystaceus & Keban Dam Lake & Sağlam (1992) \\
\hline \multicolumn{3}{|l|}{ Piscicola sp. } \\
\hline Luciobarbus pectoralis & Keban Dam Lake & $\begin{array}{l}\text { Özdemir and Sarieyyüboğlu } \\
\text { (1993) }\end{array}$ \\
\hline
\end{tabular}


Although parasite at species and genera level were reported from freshwater fish, only the species level will be considered here (Tab. 3). Other parasites at genera level (unnamed species) will be omitted in here.

Because they are reported from different hosts, the fish that will be shown (Tab. 5) may be a new species or the same species.

This revision presents an occurence of 60 monogenean at species level and eight genera level (unnamed), after eleven years (Tab. 3). Other eight unnamed monogenea (genera level) reported from different host fish (Ancyrocephalus sp., Paradiplozoon sp., Cleidodiscus sp., Diplozoon sp., Dactylogyrus sp., Gyrodactylus sp., Salsuginus sp., Tetraonchus sp.) (Tab. 5). Dactylogyrus and Gyrodactylus genus are the most abundant among the monogenea. Dactylogyrus genus is represented with 38 species, while Gyrodactylus with 11 species. Dactylogyrus is the dominant genus among the Monogenea with regard to species diversity, host distribution and location. 33 host fish species (32 species of Cyprinidae, one species of Siluridae) were infested with Dactylogyrus; 13 host fish species (nine species of Cyprinidae, three species of Gobiidae, one species of Gasterosteidae) with Gyrodactylus; 10 different fish species with Paradiploozon and Diplozoon. Dactylogyrus chalcalburni, Ancylodiscoides siluri, Ancylodiscoides vistulensis and Tetraonchus monenteron may be considered specific to their hosts. Dominants of some monogeneans selected the following infection sites: Dactylogyrus from gill filaments; Gyrodactylus from fins, gills, body surface.

This revision presents occurence of 20 digenean at species level and 11 digenean at genera level (unnamed), after eleven years (Tab. 3). Also, 11 unnamed species (genera level) were reported from different host fish (Ascocotyle sp., Digenea sp., Diplostomum sp., Diplodiscus sp., Neascus sp., Orientocreadium sp., Posthodiplostomum sp., Rhipidocotyle sp., Sanguinicola sp., Tetracotyle sp., Creptotrema sp.). Digenea is next-largest group with 20 species. In terms of host distribution, Digenea may be ranked as follows: Diplostomum (seven hosts), Tylodelphys (13 hosts), Clinostomum (10 hosts), Asymphylodora (four hosts), Posthodiplostomum (six hosts), Bucephalus (four hosts), Allocreadium (four hosts), Aspidogaster (five hosts). Bucephalus polymorphus may be cosidered specific to carnivorous fish. Adults digeneans were reported from intestine, pyloric caeca, stomach, body cavity, eye lens, liver, spleen, pericard, gall bladder, heart of hosts. Larvaes of some digeneans such as Centrocestus sp., Clinostomum sp., Posthodiplostomum sp., were reported from fins, body surface, operculum, muscle, gills of hosts.

The checklist contains 20 species and eight unnamed species (genera level) of Cestoda. In terms of host distribution, the 10 genera of Cestoda may be ranked as follows: Ligula (28 hosts), Bothriocephalus (17 hosts), Caryophyllaeus (11 hosts), Proteocephalus (four hosts) Khawia (six hosts). Ligula pavlovskii, Triaenophorus crassus, Silurotaenia siluri, Khawia armeniaca and Proteocephalus osculatus may be considered specific to their hosts. Ligula intestinalis is the dominant cestode species in terms of host range and location. Cestodes were reported from intestine, body cavity, surfaces of visceral organs, pharynx, external mesenteries of internal organ, pyloric caeca, duodenum, urogenital ducts, liver, gonad of hosts.

The checklist contains 11 species and nine unnamed species (genera level) of Nematoda. In terms of host distribution, the genera of Nematoda may be ranked as follows: Rhabdochona (eight hosts), Eustrongylides (nine hosts), Philometra (three hosts). Adults nematodes were reported from body cavity, pyloric caeca, liver, intestine, mesentery, body surface, gills, coelom, stomach, swimbladder, surfaces of visceral organs, muscle, ovarium, testis of hosts. Nematoda sp. larvae were reported from body surface, gills. 
The checklist contains seven species and four unnamed species (genera level) of acanthocephala. The genera of Acanthocephala are distributed as follows: Neoechinorhynchus (15 hosts), Pomphorhynchus (14 hosts), Acanthocephalus (two hosts). Acanthocephalans were only reported on intestines of hosts.

The checklist contains five species and two unnamed species (genera level) of hirudinids. Hirudinids were reported on body surface, fins, gills, mouth.

After the checklist of helminths of freshwater fish from Turkey published by Öktener (2003), parasites species number increased significantly to nowadays. Especially, platyhelminth species number reached from 57 to 100 (Tab. 6).

Table 6: Change of number of named and unnamed helminth species reported from fish species occurring after Öktener (2003).

\begin{tabular}{|l|c|c|c|c|}
\hline & \multicolumn{2}{|c|}{$\begin{array}{c}\text { Öktener } \\
\text { (2003) }\end{array}$} & \multicolumn{2}{c|}{$\begin{array}{c}\text { Present } \\
\text { Study }\end{array}$} \\
\hline & $\begin{array}{c}\text { Named } \\
\text { species }\end{array}$ & $\begin{array}{c}\text { Unnamed } \\
\text { species }\end{array}$ & $\begin{array}{c}\text { Named } \\
\text { species }\end{array}$ & $\begin{array}{c}\text { Unnamed } \\
\text { species }\end{array}$ \\
\hline Monogenea & 28 & 5 & 60 & 8 \\
\hline Digenea & 16 & 5 & 20 & 11 \\
\hline Cestoda & 13 & 6 & 20 & 8 \\
\hline Nematoda & 8 & 5 & 11 & 9 \\
\hline Acanthocephala & 5 & 3 & 7 & 4 \\
\hline Hirudinea & 3 & 2 & 5 & 2 \\
\hline & 73 & 26 & 123 & 42 \\
\hline Total & 5 & 99 & \multicolumn{2}{c|}{165} \\
\hline
\end{tabular}

\section{CONCLUSIONS}

This new updated checklist is done to update the helminths of freshwater fish from Turkey.

Finally, it was also planned to show and update the parasite richness of fish of Turkey according to actual literature.

The present publication describes the published literature and thus provides a summary of the currently known freshwater fish parasites in Turkey. It was felt that a critical checklist of the freshwater fish parasites known from Turkey to date would help to solve contradictions among researchers, and benefit veterinarians, parasitologists and zoologists, ecologists.

It is hoped that this compilation will stimulate further parasitological investigations of fish in Turkey. 


\section{REFERENCES}

1. Akbeniz E., 2006 - Metazoon parasites of tench (Tinca tinca Linnaeus, 1758) in Sapanca Lake, Marmara University, Institute of Science, MSc Thesis, 47.

2. Akinci A. G., 1999 - Studies On Determine The Platyhelminths of Blicca bjoerkna L., 1758 in Uluabat Lake, Uludağ University, Institute of Science, MSc Thesis, 34.

3. Akmirza A., 2007 - The effect of Ligula intestinalis L. plerocercoid on the growth of bitterling (Rhodeus amarus Bloch, 1782), Journal of the Black Sea/Mediterranean Environment, 13, 155160.

4. Aksakal H. N., 1992 - The Parasitological Studies On the Identification of Platyhelminthes Living As A Endoparasites in Tenchs (Tinca tinca) of Uluabat Lake, Uludağ University, Institute of Science, MSc Thesis, 24.

5. Aksoy Ş., 1996 - Endohelminths research in Capoeta capoeta umbla from Hazar Lake, Firat University, Institute of Science, MSc Thesis, 39.

6. Aksoy Ş., Sağlam N. and Dörücü M., 2006 - External Parasites of Three Cyprinid Fish Species from Lake Hazar in Turkey, Indian Veterinary Journal, 83, 100-101.

7. Altin H., 1989 - Some Parasites On Carp (Cyprinus carpio L.), Uludağ University, Science Faculty, BSc Thesis, 33.

8. Arthur J. R. and Ahmet A. T. A., 2002 - Checklist of the parasites of fishes of Bangladesh, FAO Fisheries Technical Paper, 369, 1, Rome, 77.

9. Arthur J. R. and Lumanlan-Mayo S., 1997 - Checklist of the parasites of fishes of the Philippines, FAO Fisheries Technical Paper, 369, Rome, 102.

10. Aslan B., 2009 - Investigations of the endohelminths of some fish from Murat River (Ağri) and Aras River (Erzurum), Atatürk University, Science Institution, Institute of Science, 58.

11. Atay D., Köksal G., Seçer S., Aydin F., Polatsü S. and Yildiz H., 1999 - Impact on the Health Status and some Hematologic Characteristics of Carp Fertilization, Turkish Journal of Zoology, 20, 67-72.

12. Aydin Y., 2003 - The Helminths of the Digestive Tract of Catfish (Silurus glanis L., 1758) in Hirfanli Dam Lake, Nigde University, Institute of Science, MSc Thesis, 50.

13. Aydoğdu A., 1997 - Studies on determine the platyhelminth parasites of common carp (Cyprinus carpio L., 1758) in İznik Lake, Uludağ University, Institute of Science, MSc Thesis, 35.

14. Aydoğdu A., 2001 - The Helminthofauna of Some Fishes Living in Doganci Dam Lake, Uludag University, Institute of Science, PhD Thesis, 82.

15. Aydoğdu A., Emence H. and Altunel A., 2008a - Helminth parasites of pike (Esox lucius L.) in Gölbaşi (Bursa) Dam Lake, Turkey, Pakistan Journal of Zoology, 40, 3, 221-224.

16. Aydoğdu A., Emence H. and İnnal D., 2008b - The Occurrence of Helminth Parasites in Vimba (Vimba vimba L., 1758) of Golbasi (Bursa) Dam Lake, Turkey, The Turkish Journal of Parasitology, 32, 1, 86-90.

17. Aydoğdu A., Emre Y., Emre N. and Altunel F. N., 2011 - The occurrence of helminth parasites (Nemathelminthes) in some freshwater fish from streams discharging into Antalya Bay in Antalya, Turkey: two new host records from Antalya, Turkish Journal of Zoology, 35, 6, 859864.

18. Aydoğdu A., Oğuz M. C., Öztürk M. O. and Altunel F. N., 2001 - Investigations on metazoon parasites of common carp (Cyprinus carpio L., 1758) in Dalyan Lagoon, Karacabey, Acta Veterinaria, 51, 5-6, 351-358.

19. Aydoğdu A. and Selver M., 2006 - An Investigation of Helminth Fauna of the Bleak (Alburnus alburnus L.) from the Mustafakemalpasa Stream, Bursa, Turkey, The Turkish Journal of Parasitology, 30, 1, 69-72.

20. Aydoğdu A., Yildirimhan H. S. and Altunel F. N., 1996 - An Investigation On Parasites of Catfish (Silurus glanis L., 1758), in Iznik Lake, XIII, National Biology Congress (17-20 September, 1996), Istanbul, 63-70. 
21. Aydoğdu A., Yildirimhan H. S. and Altunel F. N., 1996a - An investigation on parasites of tench (Tinca tinca L., 1758) in the İznik Lake, The Turkish Journal of Parasitology, 20, 261270.

22. Aydoğdu A., Yildirimhan H. S. and Altunel F. N., 1997b - An investigation on ecto and endo helminth of roach (Rutilus frisii L.), IX National Aquatic Products Symposium (17-19 September 1997), Isparta, 431-443.

23. Aydoğdu A., Yildirimhan H. S. and Altunel F. N., 1997 - An investigation on some metazoan parasites of common carp (Cyprinus carpio L.) in İznik Lake, The Turkish Journal of Parasitology, 21, 4, 442-445.

24. Aydoğdu A., Yildirimhan H. S. and Altunel F. N., 2000 - The Helminth Fauna of Adriatic Roach (Rutilus rubilio) in Iznik Lake, Bulletin of European Fish Pathology, 20, 3, 170-172.

25. Başaran A. and Kelle A., 1976 - Distribution of Ligula intestinalis in Some Freshwater Fish Living On the Devegecidi Dam Lake, Journal of Hacettepe University, 26, 45-56.

26. Becer Z. A. and Kara D., 1998 - An investigation on population structure and parasites of common carp (Cyprinus carpio L., 1758) which were caught in Kovada Lake, The Turkish Journal of Parasitology, 22, 2, 199-203.

27. Buhurcu H. İ., 2006 - An investigation on endoparasite fauna of some fish species (Cyprinus carpio and Alburnus nasreddini) from Lake Akşehir, Afyon Kocatepe University, Institute of Science, MSc Thesis, 42.

28. Bulgen K., 1999 - An investigation of the taxonomic status and biological aspects of the chub (Leuciscus cephalus Linnaeus, 1758), Balikesir University, Institute of Science, MSc Thesis, 48.

29. Burgu A., Oğuz T., Körting W. and Güralp N., 1988 - Parasites of freshwater fishes in some areas of central Anatolia, Journal of Etlik Veterinary and Microbiology, 3, 6, 143-165.

30. Caira J. N., 1989 - A revision of the North American papillose Allocreadiidae (Digenea) with independent cladistic analyses of larval and adult forms, Bulletin of the University of Nebraska State Museum, Lincoln, 11, 3, 1-58.

31. Cantoray R. and Özcan A., 1975 - La ligulose chez des poissons d'eau douce aux alentours d’Elaziğ, Firat University, Journal of Veterinary Faculty, 2, 298-301. (in French)

32. Cengizler İ., Sarihan E. and Çevik C., 1991 - Investigation of Ligulosis on cyprinids in Almus Lake, Symposium of National Aquatic Products, 12-14 Kasim 1991, İzmir, 371-375.

33. Cengizler I. and Göksu L., 1994 - Some Metazoon Parasites Seen in Two Cyprinid Species Lived In Balikliag Stream, XII National Biology Congress (6-8 July 1994, Edirne), Edirne, 362-365.

34. Cengizler İ., Aytaç N., Şahan A., Özak A. A. and Genç E., 2001 - Ecto-Endo Parasite Investigation on Mirror Carp (Cyprinus carpio L., 1758) Captured From the River Seyhan, Turkey, Ege University, Journal of Fisheries and Aquatic Sciences, 18, 1-2, 87-90.

35. Ceylan Y., 2002 - Parasitological Research of fishes in Çapali Lake (Dinar-Afyon), Süleyman Demirel University, Institute of Science, MSc Thesis, 54.

36. Ceylan M., Boyaci Y. E., Meke T., İnceoğlu H. and Kara A., 2011 - A Report of Ectoparasite Piscicola geometra (Linnaeus, 1761) (Hirudinea: Rhynchobdellida) on Roach (Rutilus rutilus (Linnaeus, 1758)) from Uluabat Lake, The Turkish Journal of Parasitology, 35, 207-209.

37. Çolak H. S., 2013 - Metazoan parasites of fish species from Lake Siğirci (Edirne, Turkey), Turkish Journal Veterinary Animal Science, 37, 200-205.

38. Çolak S. Ö., 2013 -The helminth community of the sand smelt (Atherina boyeri Risso, 1810) from Lake Iznik, Turkey, Journal of Helminthology, 87, 129-134.

39. Dal A., 2006 - Parasitological research on rainbow trout (Oncorhynchus mykiss) farming in Atatürk Dam Lake, Çukurova University, Institute of Science, MSc Thesis, 52.

40. Develi N., 2008 - Seasonal Distribution of metazoan parasites in fish species found in Almus Dam Lake, Gaziosmanpaşa University, Institute of Science, MSc Thesis, 65. 
41. Diler Ö., Yildirim U. G., Küçük F. and Işikli B., 2001 - Parasitological research on cobitid (Knipowitshia caucasica) living in Eğirdir Lake, 11 Symposiyum of National Aquatic Products, Hatay 04-06 Eylül, 2001.

42. Dörücü M., Kan N. İ. and Öztekin Z., 2008 - Investigation of internal parasites of some fish species caught in Keban Dam Lake (Turkey), Journal of Fisheries Sciences, 2, 3, 484-488.

43. Dörücü M. and İspir Ü., 2001 - Seasonal Variation of Diplostomum sp. Infection in Eyes of Acanthobrama marmid Heckel, 1843 in Keban Dam Lake, Elaziğ, Turkey, Ege University, Journal of Fisheries and Aquatic Sciences, 18, 3-4, 301-305.

44. Dörücü M. and İspir Ü., 2005 - A Study on Endo-Parasites of Some Fish Species Caught in Keban Dam Lake, Journal of Firat University Science and Engineering, 17, 2, 400-404.

45. Ekingen G., 1975 - Some parasites found on brown trout (Salmo trutta L.) in Munzur Stream, Journal of Firat University Veterinary Faculty, 2, 283-301.

46. Ekingen G., 1976 - Some parasites found european catfish (Silurus glanis L.) and brown trout (Salmo trutta L.) in Turkey, Journal of Firat University Veterinary Faculty, 3, 112-115.

47. Ekmekçi F. G., 1989 - Examination of fish stocks have economic importance in Sariyar Dam Lake, Hacettepe University, Institute of Science, PhD Thesis, 114.

48. Ekmekçi F. G. and Kirankaya Ş. G., 2004 - Determination of Variations in Fish Growth during Reservoir Ontogeny: a Case Study of the Mirror Carp Population in Geling, ll, Dam Lake (Yozgat, Turkey), Turkish Journal of Veterinary Animal Science, 28, 1129-1135.

49. Emiroğlu Ö. and Arslan N., 2009 - First Record of Parasitic Annelida-Hirudinea (Piscicola geometra Linnaeus, 1761) on Carassius gibelio from Lake Uluabat, 11th International Symposium on Aquatic Oligochaeta 2009, Alanya, Türkiye, 62.

50. Erkul S., 1997 - Infection of helminths in freshwater fish seen in the region of Ankara, Ankara University, Institute of Health Science, MSc Thesis, 48.

51. Eschmeyer W. N. (ed.), 2014 - Catalog of fishes: genera, species, references, (http://research.calacademy.org/research/ichthyology/catalog/fishcatmain.asp), Electronic version (Accessed 13.02.2014).

52. Froese R. and Pauly D. (eds), 2013 - FishBase, World Wide Web electronic publication, www.fishbase.org, Electronic version (Accessed 06.03.2014).

53. Geldiay R. and Balik S., 1974 - Ecto and Endoparasites Found the Freshwater Fish of Turkey, Ege University, The Science Faculty Monographies, 14, Ege University Press, Bornova.

54. Gibson D. I., Bray R. A. and Harris E. A., 2005 - Host-Parasite Database of the Natural History Museum, London, available from: http://www.nhm.ac.uk/research-curation/scientificresources/taxonomy-sytematics/host-parasites (Accessed 06.03.2014).

55. Güleç A. K. and Şahan A., 2010 - Effects to Plasma Glucose, Cortisol and Haemoglobin Levels of Parasite Enfestations in Carp (Cyprinus carpio Linnaeus, 1758), Kafkas University, Journal of Science, 3, 1, 1-8.

56. Gürkan Ü. and Özan S. T., 2012 - Helminth fauna of chub (Squalis cephalus L.) in Susurluk Creek (Bursa-Balikesir), Süleyman Demirel University, Journal of Science, 7, 2, 77-85.

57. Holland C. V. and Kennedy C. R., 1997 - A checklist of parasitic helminth and crustacean species recorded in freshwater fish from Ireland, Biology and Environment: Proceedings of the Royal Irish Academy, 97B, 3, 225-243.

58. ITIS, 2013 - The Integrated Taxonomic Information System, http://www.itis.gov., University, Institute of Science, MSc Thesis, 103.

59. İnnal D., 2004 - Stock assesment of some fish species living in Çamkoru Pond (ÇamlidereAnkara), Hacettepe.

60. İnnal D. and Keskin N., 2005 - Philometra ovata (Zeder, 1803) (Philometridae) in European Chub (Leuciscus cephalus L., 1758) living in Çamkoru Lake (Çamlidere), Journal of Animal and Veterinary Advances, 4, 12, 959-961.

61. İnnal D., Keskin N. and Erk'akan F., 2007 - Distribution of Ligula intestinalis (L.) in Turkey, Turkish Journal of Fisheries and Aquatic Sciences, 7, 19-22. 
62. Innal D., Erk'akan F. and Keskin N., 2010 - The Dynamics of the Ligula intestinalis (Cestoda: Pseudophyllidea) in Three Cyprinid Species (Alburnus escherichii Steindachner, 1897; Gobio gobio (Linnaeus, 1758) and Squalius cephalus (Linnaeus, 1758)) in Çamkoru Pond (Ankara, Turkey), Hacettepe, Journal of Biology and Chemistry, 38, 4, 319-324.

63. Kahveci S., 2004 - The metazoon parasites of rud (Scardinius erythrophthalmus Linnaeus, 1758) caught in Lake Durusu (Terkos), Marmara University, Institute of Science, MSc Thesis, 51.

64. Kara D., 1997 - Investigation On Trematodes of Perch (Stizostedion lucioperca L., 1758) in Egirdir, Beysehir Lakes, Suleyman Demirel University, Institute of Science, MSc Thesis, 54.

65. Karabiber F. T., 2006 - Parasite fauna of roach (Rutilus rutilus Linnaeus, 1758) in the Lake Sapanca, Marmara University, Institute of Science, MSc Thesis, 53.

66. Karabulut C., 2009 - Study of endohelminths on carp (Cyprinus carpio L., 1758) caught from four different regions of Keban Dam Lake (Koçkale, Pertek, Çemişgezek, Keban), Firat University, Institute of Science, MSc Thesis, 29.

67. Karakişi H. and Demir S., 2012 - Metazoan Parasites of the Common Carp (Cyprinus carpio L., 1758) from Tahtali Dam Lake (İzmir), The Turkish Journal of Parasitology, 36, 174-177.

68. Karatoy E., 2004 - Metazoon parasites of bream (Abramis brama Linnaeus, 1758) in Durusu (Terkos) Lake, Marmara University, Institute of Science, MSc Thesis, 55.

69. Kartal K., 2006 - An investigation on ectoparasite fauna of some fish (Cyprinus carpio and Cobitis simplicispinna) from Lake Akşehir, Afyon Kocatepe University, Institute of Science, MSc Thesis, 5.

70. Kelle A., 1978 - Les Effects de Ligula Intestinalis Sur Les Currelations de Taille Poids et Ces Character Biometriaues des Certain Poissons de Eaux Douces Acanthobrama marmid Heckel, 1843 and Chalcalburnus mossulensis Heckel, 1843, Journal Ege University Science Faculty, 7, 1, 95-108.

71. Keskin N., 1988 - Philometra abdominalis Nybelin, 1928 in Leuciscus cephalus in Turkey, Turkish Journal of Zoology, 12, 1, 70.

72. Keskin N. and Erakan F., 1987 - Ligulose in the Freshwater Fish in Turkey, Journal of Hacettepe University Science and Engineer Faculty, 8, 57-70.

73. Kiliç H., 1999 - Site selection, The case of Turkey, CIHEAM-Options Mediterraneennes, 43, 25-33.

74. Kilinçaslan M. O., 2007 - Parasitological Research on economic fishes in Yamula Dam Lake in Kayseri City, Çukurova University, Institute of Science, MSc Thesis, 42.

75. Kir I., 1998 - Investigation of Parasites of Carp (Cyprinus carpio L., 1758) and Barbus (Barbus capito pectoralis L., 1758) and goldfish (Carassius carassius L., 1758) Living in Karacaoren Dam Lake, Suleyman Demirel University, Institute of Science, PhD Thesis, 78.

76. Kir İ. and Özan S. T., 2004 - Occurrence of helminths in tench (Tinca tinca L., 1758) of Kovada (Isparta) Lake, Turkey, Bulletin of European Fish Pathology, 25, 2, 75-81.

77. Kohn A., Cohen S. C. and Salgado-Maldanado G., 2006 - Checklist of Monogenea parasites of freshwater and marine fishes, amphibians and reptiles from Mexico, Central America and Caribbean, Zootaxa, 1289, 1-114.

78. Koyun M., 2001 -The Helminthofauna of Some Fishes in Enne Dam Lake, Uludağ University, Institute of Science, PhD Thesis, 119.

79. Koyun M., 2011 - First record of Dogielius forceps (Monogenea) on Capoeta umbla (Pisces, Cyprinidae) to Turkey, from Murat River, International Journal of the Bioflux Society, 4, 4, 469-473.

80. Koyun M., Yilmaz F. and Alas A., 1999 - Effects On Alburnus alburnus of Pleurocercoids of Ligula intestinalis, X National Aquatic Products Symposium (22-24 September 1999, Adana), Adana, 156-163.

81. Kurupinar E., 2009 - An investigation on parasite fauna of chub (Leuciscus cephalus L., 1758) from Dam Lake Örenler (Afyonkarahisar), Afyon Kocatepe University, Institute of Science, MSc Thesis, 63. 
82. Kutlu H. L., 2005 - An investigation on anatomy, morphology and ecology of metazoan parasites of Cyprinus carpio Linnaeus, 1758 (common carp) from Lake Karamik (Afyonkarahisar), Afyon Kocatepe University, Institute of Science, MSc Thesis, 64.

83. Luque J. L. and Tavares L. E. R., 2007 - Checklist of Copepoda associated with fishes from Brazil, Zootaxa, 1579, 1-39.

84. Öge H. and Sarimehmetoglu H. O., 1996 - The Metacercaria of Clinostomum complanatum (Rudolphi, 1819) in Barbus plebejus escherichii (Steindachner, 1897) and Capoeta tinca (Heckel, 1843), The Turkish Journal of Parasitology, 20, 3-4, 429-437.

85. Oğuz M. C., 1991 - An investigation On the Carps (Cyprinus carpio L.) which were caught from some freshwaters of Bursa, The Turkish Journal of Parasitology, 15, 2, 103-110.

86. Oğuz M. C. and Özturk M. O., 1993 - A Parasitological Investigation On the Searching of the Endohelminth of the Rudd (Scardinius erythrophthalmus L., 1758), The Turkish Journal of Parasitology, 17, 3-4, 130-137.

87. Oğuz M. C., Öztürk M. O., Altunel F. N. and Ay A. D., 1996 - A parasitological investigation on common carp (Cyprinus carpio L., 1758) caught in Uluabat Lake, The Turkish Journal of Parasitology, 20, 1, 97-103.

88. Oğuz M. C., Amin O. M., Heckmann R. A., Tepe Y., Johargholizadeh G., Aslan B. and Malek M., 2012 - The discovery of Neoechinorhynchus zabensis (Acanthocephala: Neoechinorhynchidae) from cyprinid fishes in Turkey and Iran, with special reference to new morphological features revealed by scanning electron microscopy, Turkish Journal of Zoology 36, 6, 759 .

89. Oğuz M. C., Öztürk M. O. and Güre H., 2004 - Seasonal Variation of the Plerocercoid Ligula intestinalis (L.) Observed in Roach (Rutilus rutilus, L) from the Yenice Irrigation Pond, Canakkale, Turkey, Veterinarski glasnik, 58, 1-2, 127-130.

90. Öktener A., 2003 - Checklist of Metazoon Parasites Recorded in Freshwater Fish From Turkey, Zootaxa, 394, 1-28.

91. Öktener A. and Alaş A., 2009 - A parasitological study of fish from the Ataturk Dam Lake, Turkey, Bulletin of the European Association Fish Pathologists, 29, 6, 193-197.

92. Öktener A., Trilles J. P. and Leonardos I., 2007 - Five Ectoparasites from Turkish fishes, The Turkish Journal of Parasitology, 31, 2, 154.

93. Örün İ., Dörücü M., Yazlak H. and Öztürk E., 2003 - Research on effects and helminths of fish species from Karakaya Dam Lake, İnönü University, Department of Research Projects, 15.

94. Özan S. T., 2005 - The Investigation of Heavy Metals and Parasites in Carp (Cyprinus carpio L., 1758) and Tench (Tinca tinca L., 1758) Inhabiting Beysehir Lake, Süleyman Demirel University, Institute of Science, $\mathrm{PhD}$ Thesis, 93.

95. Özan S. T. and Kir İ., 2005 - An Investigation of Parasites of Goldfish (Carassius carassius L., 1758) in Kovada Lake, The Turkish Journal of Parasitology, 29, 3, 200-203.

96. Özbek M., 2009 - Investigations on Ligula sp. infection of fishes from Dam Lake Kunduzlar (Kirka, Eskişehir), Afyon Kocatepe University, Institute of Science, MSc Thesis, 47.

97. Özdemir Y. and Sarieyyupoglu M., 1993 - Some parasites of Barbus capito pectoralis caught in Keban Dam Lake, Journal of Firat University Sceince and Engineering, 5, 2, 114-126.

98. Özer A., 1995 - A research on ectoparasites of carp (Cyprinus carpio L., 1758) breeding in Sinop Region, Ondokuz Mayis University, Institute of Science, MSc Thesis, 75.

99. Özer A., 2006 - Parasite Fauna of An Endemic Toothcarp, Aphanius chantrei Gaillard, 1895 in Sinop, Turkey, 11eme Congress International de Parasitologie Glasgow-ecosse du 6 AU 11 Juillet 2006, (a 1544).

100. Özer A., 2007 -Metazoan parasite fauna of the round goby Neogobius melanostomus Pallas, 1811 (Perciformes: Gobiidae) collected from the Black Sea coast at Sinop, Turkey, Journal of Natural History, 41, 9-12, 483-492. 
101. Özer A. and Öztürk T., 2005 - Dactylogyrus cornu Linstow, 1878 (Monogenea) Infestations on vimba (Vimba vimba tenella (Nordmann, 1840)) Caught in the inop Region of Turkey in Relation to the Host Factors, Turkish Journal of Veterinary Animal Science, 29, 11191123.

102. Özer A., Öztürk T. and Öztürk M. O., 2005 - Prevalence and intensity of Gyrodactilus arcuatus Bychowsky, 1933 (Monogenea) infestations on the three-spined stickleback, Gasterosteus aculeatus L., 1758, Turkish Journal of Veterinary Animal Science, 28, 807-812.

103. Özgül G., 2008 - Seasonal Distribution of parasites on cyprinid fishes in Almus Dam Lake, Gaziosmanpaşa University, Institute of Science, MSc Thesis, 84.

104. Özgül G. and Turgut E., 2006 - Metazoan parasites on Chondrostoma regium caught from Almus Dam Lake, Symposium of Aquaculture Students, Mayis, Muğla, 23-25.

105. Öztürk M. O., 1995 - Studies on determine the endohelminths of Pike (Esox lucius) living in Uluabat Lake, Uludağ University, Institute of Science, MSc Thesis, 53.

106. Öztürk M. O., 2000 - The Helminthofauna of Fishes of Manyas Lake, Uludağ University, Institute of Science, $\mathrm{PhD}$ thesis, 134.

107. Öztürk M. O., 2002 - Metazoan parasites of the tench (Tinca tinca L.) from lake Uluabat, Turkey, Israel Journal of Zoology, 48, 4, 285-293.

108. Öztürk M. O., 2005 - An Investigation of Metazoan Parasites of Common Carp (Cyprinus carpio L.) in Lake Eber, Afyon, Turkey, The Turkish Journal of Parasitology, 29, 3, 204-210.

109. Öztürk T., 2005 - Determination of parasite fauna of flounder, Platichthys flesus L., 1758 and toothcarp Aphanius chantrei Gaillard 1895 present in Sarkum Lagoon Lake, Sinop, Turkey, Ondokuz Mayis University, Institute of Science, PhD Thesis, 327.

110. Öztürk M. O., 2010 - A Research on Plathelminth Parasites of Carassius gibelio (Bloch, 1782) in Lake Dam Seyitler, Journal of Sciences, AKÜ, 02, 91-97.

111. Öztürk M. O., 2011 - A research on helminth fauna of three cyprinid fish species in Emre Dam Lake, Afyonkarahisar, AKÜ, Journal of Sciences, 11, 23-29.

112. Öztürk T. and Özer A., 2008 - Parasitic fauna of the toothcarp Aphanius danfordii (Boulenger, 1890) (Osteichthyes: Cyprinodontidae), an endemic fish from Sarikum Lagoon Lake in Sinop (Turkey), Journal of Fisheries Sciences.com, 2, 3, 388-402.

113. Öztürk M. O. and Bulut S., 2006 - An Investigation on the Metazoan Parasite Fauna of Cyprinus carpio L. (Common Carp) from Lake Selevir Dam (Afyonkarahisar), Science and Engineering Journal of Firat University, 18, 2, 143-149.

114. Öztürk M. O., Oğuz M. C. and Altunel F. N., 2000 - Metazoon Parasites of Pike (Esox lucius L.) From Uluabat Lake, Israel Journal of Zoology, 46, 119-130.

115. Öztürk M. O, Oguz M. C. and Aydogdu A., 2002 - An investigation of metazoon parasitic fauna of pike (Esox lucius L.) and rudd (Scardinius erytrophthalmus L.) from the Karacabey Lagoon, The Turkish Journal of Parasitology, 26, 3, 325-328.

116. Palm H. W., Klimpel S. and Bucher C., 1999 - Checklist of metazoan fish parasite of German coastal waters, Institut für Meereskunde and Christian-Albrechts-Universitat Kiel, 307, 148.

117. Paperna I., 1964 - The Metazoon Parasite Fauna of Israel Inland Water Fishes, Bamidgeh, 16, 1-2, 3-66.

118. Pazooki J. and Masoumian M., 2012 - Synopsis of the Parasites in Iranian Freshwater Fishes, Iranian Journal of Fisheries Sciences, 11, 3, 570-589.

119. Pişkin F. Ç. and Ütük A. E., 2008 - Dactylogyrus sp. and Trichodina sp. cases in carps of Sanliurfa Balikligol, Etlik Veteriner Mikrobiyoloji Derg, 19, 9-12.

120. Sağlam N., 1992 - Investigation of external parasites on fish caught in Lake Keban, Firat University, Institute of Science, MSc Thesis, 50.

121. Sağlam N. and Sarıeyyupoglu M., 2002 -Study of Neoechinorhynchus rutili (Acanthocephala) Found in Fish (Capoeta trutta), The Turkish Journal of Parasitology, 26, 3, 328-330.

122. Salgado-Maldanado G., 2006 - Checklist of helminth parasites of freshwater fishes from Mexico, Zootaxa, 1324, 1-357. 
123. Samanci İ., 2011 - The investigation of parasites in carp (Cyprinus carpio L., 1758) and crucian carp (Carassius carassius L., 1758) inhabiting Karacaören II, Dam Lake, Suleyman Demirel University, Institute of Science, MSc Thesis, 46.

124. Saygi G. and Bardakci F., 1990 - Rhabdochona (Nematod) found in Cyprinion macrostomus and Garra rufa in Thermal Baths Called "Balikli Cermik”, The Turkish Journal of Parasitology, $14,1,95-105$.

125. Selver M. M., 2008 - Helminth Fauna Of Some Fish Species Catched From Kocadere Stream, Uludag University, Institute of Health Sciences, PhD thesis, 151.

126. Smales L. R., Aydoğdu A. and Emre Y., 2012 - Pomphorhynchidae and Quadrigyridae (Acanthocephala), including a new genus and species (Pallisentinae), from freshwater fishes, Cobitidae and Cyprinodontidae, in Turkey, Folia Parasitologica, 59, 3, 162.

127. Soylu E., 1985 - Studies of Fish diseases in Sapanca Fish Production Station, İstanbul University, College of Aquatic Products, 7, 22.

128. Soylu E., 1990 - Surveys On the Parasite Fauna of the Some Fishes In Sapanca Lake, Istanbul University, Institute of Marine Science, PhD thesis, 85.

129. Soylu E., 1991 - Pomphorhynchus laevis (Müller, 1776) (Acanthocephala) in Barbus plebejus escherichi Steindachner 1897 of (Sakarya) Büyükcoz Lake, Anadolu University, Journal of Science and Literature, 3, 2, 3.1

130. Soylu E., 2003 - A study on Metazoon parasites of tench (Tinca tinca) in the Lake Durusu (Terkos), XII Symposium of National Aquatic Products, 2-5 Eylül 2003, Elaziğ, 37.

131. Soylu E., 2005 - Metazoan Parasites of catfish (Silurus glanis, Linnaeus, 1758) from Durusu (Terkos) Lake, Journal of the Black Sea/Mediterranean Environment, 11, 225-237.

132. Soylu E., 2006 - Some metazoan parasites (Cestoda, trematoda and mollusca) of Blicca bjoerkna Linnaeus, 1758 from Sapanca Lake, Istanbul University Journal of Fisheries and Aquatic Sciences, 20, 51-62.

133. Soylu E., 2009 - Monogenean Parasites on the Gills of Some Fish Species from Lakes Sapanca and Durusu, Turkey, Ege University, Journal of Fisheries and Aquatic Sciences, 26, 4, $247-$ 251.

134. Soylu E., 2012 - Monogenean Parasites of White Bream (Blicca bjoerkna Linnaeus, 1758) in Lake Sapanca, Turkey, Kafkas University, Journal of the Faculty of Veterinary Medicine, 18, A, 23-28.

135. Soylu E., 2013 - Metazoan Parasites of Perch Perca fluviatilis L. From Lake Siğirci, Ipsala, Turkey, Pakistan Journal of Zoology, 45, 1, 47-52.

136. Soylu E. and Emre Y., 2005 - Metazoan Parasites of Clarias lazera Valenciennes, 1840 and Carassius carassius (Linnaeus, 1758) from Kepez I Hydro Electric Power Plant Loading Pond, Antalya, Turkey, Turkish Journal of Fisheries and Aquatic Sciences, 5, 113-117.

137. Soylu E. and Emre Y., 2007 - Monogenean and cestode parasites of Pseudophoxinus antalyae Bogutskaya, 1992 and Cyprinus carpio Linnaeus 1758 from Kepez Antalya, Turkey, Bulletin of European Fish Pathology, 27, 1, 23-28.

138. Sönmez Ş. N., 1996 - Investigation of parasitic fauna of fishes in Mogan Lake, Ankara University Institute of Science, MSc Thesis, 73.

139. Şahan A. and Cengizler İ., 2003 - Seyhan Nehri (Adana Kent İçi Bölgesi)'nde Yaşayan Adi Sazan (Cyprinus carpio) ve Biyikli Balik (Barbus rajanorum)'ta Bazi Hematolojik Parametrelerin Belirlenmesi, XII Ulusal Su Ürünleri Sempozyumu, 2-5 Eylül 2003, Elaziğg, 193. (in Turkish)

140. Tepe Y., Oğuz M. C., Belk M. and Özgen R., 2013 - Orientocreadium batrachoides Tubangui, 1931 (Orientocreadiidae): The only Trematode Parasite of Clarias gariepinus (Burchell, 1822) (Clariidae) from the Asi River (Turkey), The Turkish Journal of Parasitology, 37, 203-7.

141. Topçu A., 1993 - The Helminths of the Digestive Tract of the Carps (Cyprinus carpio) in Van Region, Yuzuncu Yil University, Institute of Science, PhD Thesis, 55. 
142. Torcu-Koç H., Erdoğan Z. and Coz Rakovac R., 2006 - The occurrence of Ligula intestinalis (L.) observed in chub (Leuciscus cephalus L.) from Caparlipatlak Dam lake, Ivrindi-Balikesir, Turkey, Periodicum Biologorum, 108, 2, 183-187.

143. Turgut E., 2005 - Niksar ve Almus Civarindaki Balik Çiftlikleri ile Doğal Ortamdaki Balik Parazitlerinin Su Kalitesi ve Mevsimlere Bağli Olarak Değişimi, Araştirma Projesi, Gaziosmanpaşa Üniversitesi, Almus Meslek Yüksekokulu, Tokat.

144. Turgut E., Develi N., Yeşilayer N. and Buhan E., 2011 - Seasonal Occurrence of Ligula intestinalis Infection in Cyprinids from Almus Dam Lake, Turkey, Kahramanmaras Sutcu Imam University, Journal Of Natural Sciences, 14, 3, 9-11.

145. Türk C., 2000 - Ecology of Ligula intestinalis found in Acanthobrama marmid (Heckel, 1843) from Keban Dam Lake and research its in vitro, Firat University, Institute of Science, MSc Thesis, Elaziğ, 45.

146. Türkmen H., 1990 - Prevalence of Digestive Track Helminth Infections in Carps and Roachs in Iznik Lake, İstanbul University, Institute of Heath Sciences, PhD Thesis, 58.

147. Uzunay E., 2006 - Metazoon Parasites of carp (Cyprinus carpio Linnaeus, 1758) and vimba (Vimba vimba Linnaeus, 1758) in the Lake Sapanca, Marmara University, Institute of Science, MSc Thesis, 49.

148. Ünsal G., 2008 - Ectoparasites on some bony fishes. Sinop University, Institute of Science, MSc Thesis, 61.

149. Violante-González J., Aguirre-Macedo L. and Mendoza-Franco E. F., 2007 - A checklist of metazoan parasites of fish from Tres Palos Lagoon, Guerrero, Mexico, Parasitology Research, $102,151-161$.

150. WoRMS Editorial Board, 2014 - World Register of Marine Species, Available from http://www.marinespecies.org at VLIZ, Accessed 2014-01-26.

151. Yetim M., 1985 - The Parasites Found the Fish Consuming In Eskişehir, Anadolu University, Institute of Science, MS Thesis, 42.

152. Yildirim M. Z., Kara D. and Becer Z. A., 1996 - Studies on the Bucephalus polymorphus Baer, 1827 which were identified in the pike-perch of Eğirdir Lake, The Turkish Journal of Parasitology, 20, 1, 105-112.

153. Yildirim M. and Ünver B., 2006 - Metazoan parasites of Alburnus chalcoides in Tödürge Lake (ZaraSivas, Turkey), Journal of Applied Ichthyology, 28, 245-248.

154. Yildiz K., 2003 - Helminth Infections in Tench (Tinca tinca) from Kapulukaya Dam Lake, Turkish Journal of Veterinary and Animal Science, 27, 671-675.

155. Yildiz H. Y., Korkmaz A. Ş. and Zencir O., 2003 - The Infection of Tench (Tinca tinca) with Ligula intestinalis Plerocercoids in Lake Beysehir (Turkey), Bulletin of European Fish Pathology, 23, 5, 223-227.

156. Yilmaz F., Solak K. and Alaş A., 1996 - A research on Ligula intestinalis L. from Yukari Porsuk, 17-20, Eylül, XIII Congress of National Biology, İstanbul, 71-79.

157. Zeren A., 2008 - Parasitological investigation on some aquatic species collected from the Tigem Reservoirs (Yeniyurt, Dörtyol, Hatay), Mustafa Kemal University, Institute of Science, MSc Thesis, 66. 